\title{
Strategic Interactions, social optimality and growth
}

\author{
David Vavra \\ CERGE-EI Charles University
}

July 16, 2002

\begin{abstract}
We focus on the implications of strategic interactions among research intensive firms on the rates of growth when the number of competing firms is small and firms recognise the effects their investment decisions have on the production behaviour of their rivals. Similar to other results, we find that the mode of oligopolist competition can have a great effect on the rate of growth, but we present novel channels of these effects. More importantly and unlike standard results, we show that for the Cournot type of competition, a (constrained) socially optimal rate of growth can be attained. On the other hand, this can never be achieved under the more severe Bertrand competition. These findings are thus parallel to the conclusions in strategic trade literature. Our results demonstrate that conclusions from the 'strategic trade' literature are robust enough, if extended into an explicitly dynamic general equilibrium framework. More importantly, though, they make evident that the questions of optimal policy design related to organisational structure and strategic conduct are tractable in the framework of endogenous growth models in sufficient detail.
\end{abstract}

\section{Introduction}

The seminal achievements of the early 1990s in the area of endogenous growth

(Grossman and Helpman, 1990a,b, and Romer, 1990a,b) have greatly contributed to our understanding of economic development and growth of nations.

In particular, they revealed important links through which the integration of the world economy and international trade may influence a country's growth prospects. All of these important contributions highlighted the significance of 
scale technology and the imperfect mode of competition. In such a setting, the engine of growth was ascribed to private incentives, unlike deus ex machina forces in previous growth literature.

The failure of the earlier literature to account for private incentives behind growth was remedied by introducing economies internal to firms. Typically these firms, whose products are vertically differentiated, engage in $R \& D$ activities that have a positive impact on the level of technology as a production factor. The introduction of technology with internal returns shifted attention to the structure of private incentives, because it simultaneously raised the issue of the organisational structure of the economy, i.e. more narrowly, the nature of competition.

No matter how distinct these issues may appear, they are nevertheless closely related. As Romer (1990b) observes, no firm facing a downward sloping average cost curve can survive pricing at marginal cost. If the firm is to be viable, it must enjoy some sort of monopoly power. He continues by arguing that the problem is not in marginal cost pricing, but rather in the price-taking behaviour assumed by perfect competition per se. In fact, monopolistic competition can be as close to marginal cost pricing as the average cost curves are close to marginal ones, yet, it is a necessary analytical tool that allows the increasing returns to scale technology to be operational (Krugman 1995).

Although an imperfectly competitive environment enable the analysis of how private motivation affects growth, many channels of this vehicle remain unexplored. As Baldwin (1998) in his survey asserts, the treatment of firms continues 
to be largely atomistic. Many authors (e.g. Grossman and Helpman, 1990a,b, and, Rivera-Batiz and Romer, 1991a,b) introduce a monopolistically competitive environment in the models of vertical differentiation with the Dixit-Stiglitz (1977) large number of firms approximation. Such treatment of oligopolist competition disregards the possibility of sophisticated decision-making taking place on a firm level. Vertically differentiated firms in their competition on product markets disregard the adverse effects their actions may have on the behaviour of their rivals. Such adverse effects are likely to arise from decisions about pricing policies as well as investment in R\&D. In addition, the mode of competition in product markets is an important factor for these effects.

Thus, the design of monopolistic competition in most endogenous growth literature makes it impossible to explore many important incentive schemes that might shape a country's development. For instance, it is believed that multinational enterprises (MNEs) are crucial to a country's growth performance, although we still know little about how this channel might operate, how these firms interact, what the key considerations are that make the firm invest abroad and what the potentially adverse effects are of such an activity, or how indigenous firms react to it. This is the case despite the fact that the fields of international trade and industrial organisation recognise many approaches of modelling such issues (Krugman, 1995). As a result, many important policy related questions continue to go unanswered. For instance, the nature of competition plays a prominent role in strategic trade literature when the optimal level and form of government intervention towards R\&D is sought, but is scarce in questions ad- 
dressed by endogenous growth models. This seems especially puzzling, as R\&D is the channel of sustained growth for many of them. Indeed, theorists involved in strategic trade literature recognise this failure of endogenous growth models, but consider them to be inappropriate for optimal policy design. In the words of Neary (1998):

One branch of the field which considers $R \& D$ in detail is the recent work on endogenous growth. However, the assumptions made about industrial structure and firm behaviour in that literature seem unattractive in the context of discussions of policy design. R\&D is often assumed to be carried out by different firms from those engaged in production. Moreover, notwithstanding the complexities of the models in other respects, equilibrium is typically assumed to be monopolistically competitive, so firms do not engage in strategic behaviour and entry into industry is free. For many purposes these assumptions are not a drawback. However, for designing optimal policies towards R\&D they seem less appropriate.

There have been few attempts in the literature to address growth-related issues in the market structure and strategic interactions of firms. Van de Klundert and Smulders (1997, henceforth denoted as K-S) and Smulders and van de Klundert (1995) are among the few who do not resort to the large number of firms approximation of Dixit and Stiglitz, and who openly consider static interactions among firms in product markets. As Yang and Heidra (1993) demonstrate, more insight can be gained from studying monopolistic competition of 
the Dixit-Stiglitz type if the number of firms is in fact small. The microeconomics of interactions on the firm level then gets more complex, because the firms behave strategically in the sense that they recognise the effects of their actions on the behaviour of their rivals in a simple static game theoretical setting. K-S treatment of this oligopolist set-up enables exploration of various growth-related issues according to the mode of competition in product markets: Bertrand and Cournot in a unifying manner. Yet, their analysis is confined to strategic interactions in the product markets and disregards the possibility of interactions between investment and production decisions. Therefore, many policy questions related to R\&D cannot be analysed with sufficient complexity.

The present paper belongs into this strand of literature. It aims at extending the framework of K-S to account for strategic interactions of firms between investment and production (pricing) decisions, without recourse to a differential game approach. We do so in a standard endogenous growth model of horizontal differentiation with accumulation of cost-reducing, firm-specific knowledge. In this setting, we consider some of the adverse effects a firm's decision about its R\&D expenditure may have on another firm's profits and behaviour during that period.

These adverse effects may occur through two channels. First, decisions about research investment influence the cost schedule of rival firms (and hence their pricing decisions) through knowledge spill-overs. Second, even without knowledge spill-overs, the reduction of costs in one firm has an impact on the pricing decisions of other firms in a static Nash equilibrium. Typically, the first channel 
(see, e.g. Smulders and van de Klundert, 1995) ignores these adverse effects and assume firms are myopic in this sense. In models of vertical differentiation, this assumption is often advocated as an approximation, if (i) knowledge spillovers are small, (ii) product types are well differentiated. The second channel is ignored altogether by assuming symmetric equilibrium in production/pricing actions unaffected by investment decisions.

The spill-over effect is generally known and relies solely on the non-rival nature of the R\&D knowledge stock. We concentrate instead on the second channel, since we feel that its approximation may conceal other potentially important channels through which strategic interactions of firms affect growth and welfare. For instance, K-S find that oligopolist pricing always leads to under-investment in firm-specific knowledge, irrespective of the mode of competition, essentially because it distorts relative prices in the economy in favour of non-technologically intensive goods. However, we show that a more careful consideration of static strategic effects creates an additional externality that may (depending on the mode of competition) enhance the motivation to invest, in a strategic manner, in order to increase profits and market share through the reaction of rivals. We demonstrate that this externality can not only offset the distortion created by relative prices, but potentially other externalities as well ${ }^{1}$.

\footnotetext{
${ }^{1}$ Although our model assumes that the core of the knowledge is firm-specific, we allow for external returns to knowledge accumulation, not appropriable by private decision makers. The negative externality presented by non-appropriability makes the market rate of growth fall short of that chosen by an idealised social planner, unless it is offset by another positive externality or removed by subsidies or taxes. The models based on "creative destruction", for instance, usually display a type of positive externality that may entirely counterweight the negative knowledge spill-over effect. This effect is based on the fact that the innovating firm does not internalise the negative effect it may have on another firm's innovation. For that reason, it is called "business stealing" externality. On the other hand, the common result in models of horizontal differentiation is that taxes and subsidies are required to restore the
} 
These findings are thus similar to conclusions in strategic trade literature where firms are engaged in a generic partial equilibrium two stage game: investing in cost reducing technology in the first stage and producing (or pricing) in the other. The common result is that firms tend to under-invest from the social (cost-minimising) perspective in Bertrand and over-invest in the Cournot (Brander, 1995) mode of competition. Our results demonstrate that conclusions from this strategic trade literature are robust if extended into an explicitly dynamic general equilibrium framework. More importantly, though, the results make it evident that questions of optimal policy design related to organisational structure and strategic conduct are tractable in the framework of endogenous growth models in sufficient riskness.

Similar in spirit to our paper is the work of Peretto (1996), who also recognises the need to analyse the interactions of firms with in-house R\&D in oligopolist competition. His theoretical game framework allows for the interaction of incentives from investment to pricing decisions through knowledge spill-overs. Yet, his analysis lacks an important channel of these interactions by assuming a symmetric equilibrium in product markets not affected by investment decisions. Nor does he explicitly consider different assumptions about market structure the way K-S do.

Even though we extend the K-S framework to more complex strategic conduct, our treatment of the channels through which strategic behaviour affects growth is not exhaustive. We disregard, for instance, the strategic interactions social optimum, as shown in K-S. 
of investment decisions in different periods. This is a simplification, because even if the core of the knowledge is firm-specific, firms still benefit from their rival's research. As standard in the literature, we treat these eventual spill-overs as unintentional and thus disregard the possibility that today's R\&D investment affects the rival's profits in future periods. Nevertheless, yet more insight about the channels through which interactions among firms affect growth could be gained from considering the firms' decision making in an explicitly dynamic game setting, an approach commonly referred to as differential games. However, this strand of literature suffers from great computational difficulties, which seriously constrain functional forms if the closed form solutions are to be analytically tractable. Hence, we do not pursue this here, although Vencatachellum (1998) shows the capacity of such a treatment using a modified version of Romer's 1990 model.

In the next two sections, we introduce a basic endogenous growth model with firm-specific knowledge that is a variant to that of van de Klundert and Smulders (1997) and characterise its equilibrium. We chose to follow this model, because by avoiding unnecessary deviations, we may directly compare the results and extract the novel effects of our exposition in a clear way. In the third section, we study how these extra channels modify the traditional effects of competition on growth rates and how the market equilibrium compares to the one chosen by an idealised social planner. As expected, the standard results in the literature are special cases of ours when the part of strategic decision making which is a subject of this paper is ignored. In the fourth section, we investigate whether 
strategic interactions alter the traditional result that growth rises with higher concentration. The last section presents the conclusions.

\section{Model}

Because our general equilibrium model is essentially a standard one (see e.g. van de Klundert and Smulders, 1997), we leave out most of the commentary and concentrate on developing the basic results. This is best accomplished by independently considering the private decisions and incentives of households, R\&D firms, and traditional firms, and finally characterising the market and social equilibria.

\subsection{Households}

There are a total of $L$ households in the economy who own all production units and supply them with a unit of labour as the only factor of production. In each period, households consume two types of goods: a traditional one and $N$ varieties of technology intensive (hi-tech) goods. They trade off future for current consumption according to an intertemporal utility function of the form:

$$
U=\int_{0}^{\infty} e^{-\theta t} \ln \left(C_{t}\right) d t
$$

where $\theta$ is the rate of time preference and $C$ is a consumption bundle consisting of a traditional good $Y$, and a bundle of $N$ imperfectly substitutable hi-tech goods $X$, tied together by preferences of the form:

$$
C=X^{\sigma} Y^{1-\sigma}, X=\left(\sum_{j=1}^{N} x_{j}^{\frac{\epsilon-1}{\epsilon}}\right)^{\frac{\epsilon}{\epsilon-1}}, \epsilon>1, \sigma \in(0,1),
$$


where $\sigma$, the elasticity of intratemporal substitution between $\mathrm{Y}$ and $\mathrm{X}$, is the share of hi-tech goods in the overall expenditure ${ }^{2}$. Since the utility function is weakly separable in subutilities $C$ and $X$, we may treat them as composite consumption bundles and assign to them relevant composite price indices $P_{C}$ and $P_{X}$, which correspond to the unit expenditure functions necessary for achieving a unit of subutility $C$ and $X$. Hence these price indices are given as

$$
P_{C}=\left(\frac{P_{Y}}{1-\sigma}\right)^{1-\sigma}\left(\frac{P_{X}}{\sigma}\right)^{\sigma} \text { and } P_{X}=\frac{1}{\left(\sum_{j=1}^{N} p_{i}^{1-\epsilon}\right)^{1 /(\epsilon-1)}},
$$

where $P_{Y}$ and $\left\{p_{i}\right\}_{i=1}^{N}$ are prices of $\mathrm{Y}$ and $\mathrm{X}$ goods faced by households in each $\operatorname{period}^{3}$.

This separation enables us to examine a representative households decision making in three steps: the intertemporal problem of choosing the time path of $C$, the intratemporal problem of allocating $C$ into $X$ and $Y$, and the intratemporal problem of allocating $X$ into $\left\{x_{i}\right\}_{i=1}^{N}$.

\subsubsection{The intertemporal problem}

Choosing an optimal path of $C$ in (1) is equivalent to choosing a path of total expenditure on all types of goods $E_{C}=P_{C} C$ in a modified problem:

$$
\max _{\left\{E_{t}, S_{t}\right\}_{t=0}^{\infty}} U=\int_{0}^{\infty} e^{-\theta t} \ln \left(\frac{E_{C, t}}{P_{C, t}}\right) d t, \text { s.t. } \dot{S}_{t}=Y_{t}+r_{t} S_{t}-E_{C, t},
$$

where $S_{t}$ is the total amount of saving at period $t, r_{t}$ is the period's interest rate and $Y_{t}$ is the household's income from factor ownership, treated in the

\footnotetext{
${ }^{2}$ In terms of the van de Klundert and Smulders paper, we choose the degree of risk aversion $\rho=1$. Their results are therefore comparable to ours only with this substitution.

${ }^{3}$ For brevity we suppress time indices in the following text where they are unambiguous.
} 
problem as exogenous, but consisting of labour income and distributed profits of $\mathrm{X}$ and $\mathrm{Y}$ firms net of interest payments. It is given as follows for the total of $L$ households:

$$
Y=w L+\sum_{j=1}^{N} \pi_{i}+\pi_{Y}-r S,
$$

where $w$ is the wage rate of homogenous labour and symbols $\pi_{i}, \pi_{Y}$ denote per period profits of a representative hi-tech firm, and the total of traditional firms.

From the standard solution to the problem in (3), it follows that the optimal path of expenditure must satisfy the following condition:

$$
\frac{\dot{E}_{C, t}}{E_{C, t}}=r_{t}-\theta .
$$

The necessary condition for the time path of $C_{t}$ can be readily obtained from condition (5) and price formula (2).

\subsubsection{The intratemporal problem of choosing $\mathrm{X}$ and $\mathrm{Y}$}

Given the time path of total expenditure in (5), consumers allocate this expenditure in every period between good $\mathrm{Y}$ and a bundle of hi-tech goods $\mathrm{X}$, given the prices $P_{Y}$ and $P_{X}$. The demand functions follow from the necessary conditions of optimal allocation:

$$
X=\frac{E_{C} \sigma}{P_{X}} \text { and } Y=\frac{E_{C}(1-\sigma)}{P_{Y}} .
$$




\subsubsection{The intratemporal problem of choosing $x_{i}$}

Consumers allocate the optimal expenditure on $\mathrm{X}$ type goods, $E_{X}=P_{X} X$, from (6) among individual varieties facing prices $\left\{p_{i}\right\}_{i=1}^{N}$. The symmetric demand functions, from necessary conditions of optimality, are obtained as:

$$
x_{i}=\frac{E_{X}}{p_{i}^{\epsilon} \sum_{j=1}^{N} p_{j}^{1-\epsilon}}=\frac{E_{C} \sigma}{p_{i}^{\epsilon} \sum_{j=1}^{N} p_{j}^{1-\epsilon}}, i=1, \ldots, N .
$$

Demand functions defined as such (i.e. $\left\{x_{i}\right\}_{i=1}^{N}$ in terms of $\left\{p_{i}\right\}_{i=1}^{N}$ ) appear natural if firms choose prices when maximising their profits. If, on the other hand, firms choose quantities, the demand system (7) is better thought of as defining $\left\{p_{i}\right\}_{i=1}^{N}$ in terms of $\left\{x_{i}\right\}_{i=1}^{N}$. Inverting the system in (7), we obtain:

$$
p_{i}=\frac{E_{C} \sigma}{x_{i}^{1 / \epsilon} \sum_{j=1}^{N} x_{j}^{\frac{\epsilon-1}{\epsilon}}} .
$$

This completes the description of households behaviour.

\section{$2.2 \quad Y$ good firms}

We assume an infinite number of competitive firms producing a traditional good ${ }^{4}$ using CRS technology with the unitary unit labour requirements:

$$
Y=L_{Y},
$$

where $L_{Y}$ is the number of people employed in the traditional sector. Because of perfect competition, traditional firms will not generate any surplus over their labour cost, but will follow marginal cost pricing:

\footnotetext{
${ }^{4}$ In the original paper, this sector is described as having exhausted its learning opportunities.
} 


$$
P_{Y}=w,
$$

where $w$ is the nominal wage rate prevailing in the economy. Since only real variables can be determined in the equilibrium, we later normalise $w=1$ by a choice of units.

\section{$2.3 \quad \mathrm{X}$ good firms}

Each variety is produced using IRS technology with a fixed flow of labour, $f$, necessary to maintain production, and efficiency depending on the accumulated level of firm-specific knowledge:

$$
x_{i}=h_{i} L_{X_{i}} .
$$

$L_{x_{i}}$ is the number of people employed in firm $i$ in the production itself (i.e. manufacturers), and $h_{i}$ is the level of firm-specific knowledge that accumulates according to:

$$
\dot{h}_{i}=\gamma h_{i}^{1-\alpha} H^{\alpha} L_{I_{i}}
$$

where $L_{I_{i}}$ is the number of employees in firm $i$ involved in innovative $\mathrm{R} \& \mathrm{D}$, which improves the productivity of the workforce in production, and $H$ is the industry average level of knowledge across firms ${ }^{5}$. Hence, the total amount of labour employed by a firm is:

\footnotetext{
${ }^{5}$ The specification in (11) asserts that research productivity depends on the ratio of a firmspecific level of knowledge to an industry average, rather than on an industry-wide stock of knowledge. This is clearly seen by rewriting (11) as $\dot{h}_{i}=\gamma\left(\frac{H}{h_{i}}\right)^{\alpha} h_{i} L_{I_{i}}$. As a corollary, the average productivity in research relative to industry, $\frac{\gamma h_{i}^{1-\alpha} H^{\alpha}}{h_{i}}=\gamma\left(\frac{H}{h_{i}}\right)^{\alpha}$, is independent of
} 


$$
f+L_{X_{i}}+L_{I i}
$$

Research activity benefits from its own research base $h_{i}$ and spill-overs from an average level of knowledge in industry $H$ with diminishing returns to both. We also assume that each variety is produced by a single firm and vice versa. It will be clear from the equilibrium below that no firm would find it optimal to produce a variety already produced by another company, if it can enter its own profitable niche. Because we only analyse symmetric equilibria along the BGP, we assume that all firms start with the same level of firm-specific knowledge.

At every instant, each firm chooses a price $\left(p_{i, s}\right)$ or quantity $\left(x_{i, s}\right)$ for its production and the amount of labour devoted to research so as to maximise its net present value, not taking into account the effects of its actions on the knowledge accumulation of other firms. Under this assumption, we confine ourselves to strategic interactions at the level of the production/pricing decisions of firms for the benefit of standard dynamic optimisation techniques, unlike the differential game approach. These strategic interactions take place in two modes of competition: Bertrand (firms compete in prices), and Cournot (firms compete in quantities). According to the mode, firms solve one of the following problems:

$$
V_{i, t}^{B}=\max _{\left\{p_{i}, L_{I_{i}}, h_{i}\right\}_{t=0}^{\infty}} \int_{t}^{\infty} e^{-\int_{t}^{s} r_{\tau} d \tau} \ldots
$$

the number of competing hi-tech firms. For this reason, the specification precludes analysing the effects which a varying number of competing firms might have on the possibility of inter firm learning. See, for instance, Smulders and van de Klundert (1995) for these types of effects. 


$$
\ldots\left[x_{i, s} p_{i, s}-w_{s}\left(f+L_{X_{i}, s}+L_{I i, s}\right)\right] d s
$$

s.t. $(7),(10),(11), i=1, \ldots, N$., or

$$
\begin{aligned}
& V_{i, t}^{C}=\max _{\left\{x_{i}, L_{I_{i}}, h_{i}\right\}_{t=0}^{\infty}} \int_{t}^{\infty} e^{-\int_{t}^{s} r_{\tau} d \tau}\left[x_{i, s} p_{i, s}-w_{s}\left(f+L_{X_{i}, s}+L_{I i, s}\right)\right] d s, \\
& \text { s.t.(8), (11),(10), } i=1, \ldots, N .
\end{aligned}
$$

Because the choice of price (or quantity) has no dynamic effect between periods, each firm's actions at every instant can be thought of as taking place in two stages: In the second stage, the firm chooses its price (or quantity) given the path of its research base $h_{i, t}$ and the input to research process $L_{i, t}$ chosen in the first stage. In the first stage, the firm chooses the optimal time path of the research base by allocating labour to research, and envisaging the outcomes of the second stage. Hence the problem in 12 is equivalent to:

$$
\begin{aligned}
& \max _{\left\{L_{I_{i}}, h_{i}\right\}_{t=0}^{\infty}} \int_{t}^{\infty} e^{-\int_{t}^{s} r_{\tau} d \tau}\left[\widetilde{V}_{i}^{l}(\mathbf{h})-w L_{I i, s}\right] d s, \\
& \text { s.t. } 11, i=1, \ldots, N ., l=C, B \\
\widetilde{V}_{i}^{B}= & \max _{p_{i}}\left\{x_{i, s} p_{i, s-} w_{s}\left(f+L_{X_{i}, s}\right) \mid \text { s.t. }(7),(10), \mathbf{h}\right\} \\
\widetilde{V}_{i}^{C}= & \max _{x_{i}}\left\{x_{i, s} p_{i, s-} w_{s}\left(f+L_{X_{i}, s}\right) \mid \text { s.t. }(8),(10), \mathbf{h}\right\},
\end{aligned}
$$

where $\mathbf{h}=\left\{h_{i}\right\}_{i=1}^{N}$ denotes a vector of knowledge stocks for all hi-tech firms. This decomposition allows us to solve the two problems separately in stages. 


\subsubsection{The intratemporal problem}

The solution to the inner problem in (14) is a standard one which leads to the following pricing rules:

$$
p_{i}=\frac{w}{h_{i}} \frac{e_{i}}{e_{i}-1}
$$

where $e$ is the perceived price elasticity of output for firm $i$. The higher the perceived elasticity is, the lower the mark-up and profits are at every moment, ceteris paribus. In our context, the elasticity depends on the mode of competition. It can be shown that for the Bertrand case:

$$
e_{i}^{B}=\epsilon+\frac{(1-\epsilon) p_{i}^{1-\epsilon}}{\sum_{j=1}^{N} p_{j}^{1-\epsilon}},
$$

and for the Cournot case $^{6}$ :

$$
e_{i}^{C}=\frac{1}{\frac{1}{\epsilon}+\frac{\epsilon-1}{\epsilon} \frac{x_{i}^{\frac{\epsilon-1}{\epsilon}}}{\sum_{j=1}^{N} x_{j}^{\frac{\epsilon-1}{\epsilon}}}} .
$$

Later we will be concerned only with analysing symmetric equilibria, thus these elasticities will become independent of choice variables and will be functions of parameters only:

$$
e^{B}=\epsilon+\frac{1-\epsilon}{N}, \text { and } e^{C}=\frac{\epsilon}{1+\frac{\epsilon-1}{N}} .
$$

${ }^{6}$ It seems worthwhile to emphasise that although both are own price elasticities of demand, they differ in variables that are kept constant (which are in both cases decision variables of other firms). For Bertrand they are prices and for Cournot, quantities. Hence, a more precise general notation might look like:

$e^{B} \equiv-\epsilon\left(x_{i} ; p_{i} \mid p_{-i}\right)$ and $e^{C} \equiv-\epsilon\left(x_{i} ; p_{i} \mid x_{-i}\right)$, where subindex $-i$ denotes a vector of variables other than $i$. 
Because of their distinct effects on pricing, profits and welfare, we interpret, in line with Sutton (1991), the different modes of competition as two extremes on the scale of toughness of competition ${ }^{7}$. In the symmetric equilibria studied later, we will examine the effects of competition severity by simply substituting $e^{B}$ or $e^{C}$ from (20) in place of $e$ in (17). It is, however, too simplistic to impose such symmetry on the solution of the second (production) stage before the first (intertemporal) stage of problem (14) is solved. This is our point of departure from the conventional treatment, and from Smulders and van de Klundert's in particular.

The distinction between the two modes of competition is meaningful only when the number of competing firms is relatively small (Dixit and Stiglitz, 1977, Yang and Heidra,1993); for large $N$ the two coincide. Because different strategic interactions lie at the heart of our interest, implicit in our subsequent analysis will be the requirement for a small number of hi-tech firms, achieved either by keeping the fixed cost of entry $(f)$ large enough to sustain only a few firms in a free entry equilibrium, or by preventing free entry and keeping the number of firms exogenously small.

Before symmetry is imposed, the optimal pricing decision for Bertrand competition is given by the system of (17) and (18) for all $i=1, \ldots, N$, while the optimal quantity decision for Cournot competition is determined by the system of (8), (17) and (19) for all $i=1, \ldots, N$. Hence for Bertrand, the system defines:

\footnotetext{
${ }^{7}$ Sutton, in fact, considers the weakest competition the case of joint profit maximisation.
} 


$$
p_{i}=p_{i}(\mathbf{h})=p_{i}\left(h_{i}, \mathbf{h}_{-i}\right)
$$

where $\mathbf{h}$ denotes a vector of knowledge stocks for all firms, $\left\{h_{l}\right\}_{l=1}^{N}$, and $\mathbf{h}_{-i}$ denote a vector of knowledge stocks for all other firms except $i$. The motivation for this functional notation is the apparent functional asymmetry of $h_{i}$ and $h_{j, j \neq i}$ in $p_{i}$ given by (17), and the symmetry of $h_{l, l \neq i}$ and $h_{k, k \neq i}$ given by (18). The intratemporal operational profits resulting from this optimisation and distributed at every moment among households are given as:

$$
\begin{aligned}
\pi_{i}^{B} & =x_{i}(\mathbf{p})\left(p_{i}(\mathbf{h})-\frac{w}{h_{i}}\right)-w f \\
& =x_{i}\left(p_{i}, \mathbf{p}_{-i}\right)\left(p_{i}\left(h_{i}, \mathbf{h}_{-i}\right)-\frac{w}{h_{i}}\right)-w f
\end{aligned}
$$

where $\mathbf{p}_{-i}$ denotes a price vector of $p_{j \neq i}{ }^{8}$ and where again we made use of the functional symmetry in (7). This functional form appears cumbersome, but we find it useful for intertemporal optimisation later on. In fact, in a symmetric equilibrium, it will take on a very simple form. Employing (20) and (7), we find that under symmetry:

$$
\pi_{i}^{B}=\frac{E \sigma}{N e^{B}}-w f=\frac{E \sigma}{\epsilon(N-1)+1}-w f .
$$

Likewise, for Cournot competition, optimisation defines the system of:

$$
\begin{array}{r}
\qquad x_{i}=x_{i}(\mathbf{h})=x_{i}\left(h_{i}, \mathbf{h}_{-i}\right), \\
{ }^{8} \text { More formally } p_{-i} \equiv\left\{p_{j}\left(h_{j},\left\{h_{l}\right\}_{l=1 \neq j}^{N}\right)\right\}_{j=1 \neq i}^{N}
\end{array}
$$


and

$$
\begin{aligned}
\pi_{i}^{C} & =x_{i}(\mathbf{h})\left(p_{i}(\mathbf{x})-\frac{w}{h_{i}}\right)-w f, \\
x_{i}(\mathbf{h}) & =x_{i}\left(h_{i}, \mathbf{h}_{-i}\right) \\
p_{i}(\mathbf{x}) & =p_{i}\left(x_{i}, \mathbf{x}_{-i}\right) .
\end{aligned}
$$

In a symmetric equilibrium, profits become:

$$
\pi_{i}^{C}=\frac{E \sigma}{N e^{C}}-w f=\frac{E \sigma(N+\epsilon-1)}{N^{2} \epsilon}-w f .
$$

\subsubsection{The intertemporal problem}

With the intratemporal profits defined in (26) and (22), we may now characterise the first stage of a firm's decision making. The firm decides on its investment in research $\left(L_{I, i}\right)$ given the evolution of its knowledge base (11) and facing the path of interest rates $r_{t}$ and the average level of knowledge in industry $H_{t}$. With respect to the $H_{t}$, we assume that the firm is myopic in the sense that it does not realise the effect it has over the industry average. We assume this for computational convenience, as it is not central to the argument we pursue here. As a result, the firm does not consider the effects of its actions on its rivals' profits that work through knowledge spill-overs, but instead considers the effects its research allocation has on the pricing of its rivals:

$$
\begin{aligned}
& \max _{\left\{L_{I_{i}}, h_{i}\right\}_{t=0}^{\infty}} \int_{t}^{\infty} e^{-\int_{t}^{s} r_{\tau} d \tau}\left[\pi_{i, s}^{k}-w_{s} L_{I i, s}\right] d s, \\
& \text { s.t. }(11), i=1, \ldots, N, k=B, C .
\end{aligned}
$$


The present value Hamiltonian for this problem is:

$$
H_{i, s}=e^{-\int_{t}^{s} r_{\tau} d \tau}\left[\pi_{i, s}^{k}-w_{s} L_{I_{i}, s}\right]+\lambda_{s} \gamma h_{i, s}^{1-\alpha} H_{s}^{\alpha} L_{I_{i}, s},
$$

where $\lambda_{i}$, the co-state variable, is the present shadow value of knowledge base $h$. The necessary condition for optimal development of this shadow price can be obtained as:

$$
\lambda_{i, t}=\frac{e^{-\int_{t}^{s} r_{\tau} d \tau} w_{t}}{\gamma h_{i, t}^{1-\alpha} H_{t}^{\alpha}},
$$

irrespective of the mode of competition. From this, the shadow value evolves according to:

$$
\frac{\dot{\lambda}_{i, t}}{\lambda_{i, t}}=\frac{\dot{w}_{t}}{w_{t}}-(1-\alpha) \frac{\dot{h}_{i, t}}{h_{i, t}}-\alpha \frac{\dot{H}_{t}}{H_{t}}-r_{t} .
$$

On the other hand, the necessary condition describing the firm's indifference between investing in research or production differs according to competition.

Bertrand competition Making use of (22), we obtain the following for Bertrand competition:

$$
\begin{aligned}
& \frac{\partial H_{i}^{B}}{\partial h_{i}}= \\
& e^{-\int_{t}^{s} r_{\tau} d \tau}\left[\begin{array}{c}
\nabla x_{i}(\mathbf{p})\left(\begin{array}{c}
\frac{\partial p_{i}\left(h_{i}, \mathbf{h}_{-i}\right)}{\partial h_{i}} \\
\frac{\partial \mathbf{p}_{-i}\left(h_{i}, \mathbf{h}_{-i}\right)}{\partial h_{i}}
\end{array}\right)\left(p_{i}(\mathbf{h})-\frac{w}{h_{i}}\right)+\ldots \\
\ldots+x_{i}(\mathbf{p})\left(\frac{\partial p_{i}\left(h_{i}, \mathbf{h}_{-i}\right)}{\partial h_{i}}+\frac{w}{h_{i}^{2}}\right)
\end{array}\right]+ \\
& +\quad \lambda_{i}(1-\alpha) \gamma\left(\frac{H}{h_{i}}\right)^{\alpha} L_{I_{i}}=-\dot{\lambda}_{i}
\end{aligned}
$$


where time subscripts have been suppressed for notational convenience. $\nabla x_{i}(\mathbf{p})$ denotes the (row) vector of partial derivatives with respect to elements of $\mathbf{p}$, while $\frac{\partial \mathbf{p}_{-i}\left(h_{i}, \mathbf{h}_{-i}\right)}{\partial h_{i}}$ refers to a (column) vector of partial derivatives of elements of $\mathbf{p}_{-i}$ with respect to $h_{i}$. It is this assumption in the formulation of the first order condition where we deviate from the treatment by K-S. They assume that firms do not account for adverse effects of their own R\&D choice on other firms' behaviour through knowledge spill-overs, which is a standard treatment. In addition, they impose symmetry on human capital stock across firms before the decision about $R \& D$ is made, and firms treat this path as given in the rational expectations equilibrium. Hence, the term $\frac{\partial \mathbf{p}_{-i}\left(h_{i}, \mathbf{h}_{-i}\right)}{\partial h_{i}}$ is implicitly missing in their characterisation of the firms' behaviour ${ }^{9}$.

Substituting (31) and (32) and multiplying by $h_{i}$, we obtain the following characterisation of the first order condition:

$$
\begin{array}{ll} 
& h_{i} \nabla x_{i}(\mathbf{p})\left(\frac{\partial p_{i}\left(h_{i}, \mathbf{h}_{-i}\right)}{\left.\partial h_{i}, \mathbf{h}_{-i}\right)}\right)\left(p_{i}(\mathbf{h})-\frac{w}{h_{i}}\right)+ \\
& +h_{i} x_{i}(\mathbf{p})\left(\frac{\partial p_{i}\left(h_{i}, \mathbf{h}_{-i}\right)}{\partial h_{i}}+\frac{w}{h_{i}^{2}}\right)+ \\
+\quad & (1-\alpha) w L_{I_{i}}=\frac{w}{\gamma} \frac{h_{i}^{\alpha}}{H^{\alpha}}\left[-\frac{\dot{w}_{t}}{w_{t}}+(1-\alpha) \frac{\dot{h}_{i, t}}{h_{i, t}}+\alpha \frac{\dot{H}_{t}}{H_{t}}+r_{t}\right] .
\end{array}
$$

The expression can be simplified using the functional symmetry noted above:

${ }^{9}$ If we extended our analysis to comprise the strategic interactions working through knowledge spill-overs, we would first have to recognise that the level of firm-specific knowledge is a function of the other's research stock $h_{i}\left(h_{-i}\right)$. Then the partial derivative $\frac{\partial \mathbf{p}_{-i}\left(h_{i}, \mathbf{h}_{-i}\right)}{\partial h_{i}}$ in the first order condition would have to be replaced by the total derivative $\frac{d \mathbf{p}_{-i}\left(h_{i}, \mathbf{h}_{-i}(\mathbf{h})\right)}{d h_{i}}=$ $\frac{\partial \mathbf{p}_{-i}\left(h_{i}, \mathbf{h}_{-i}\right)}{\partial h_{i}}+\nabla \mathbf{p}_{-i}\left(\mathbf{h}_{-i}\right)\left(\frac{\partial \mathbf{h}_{-i}}{\partial h_{i}}\right)$. Noting that the cross derivative $\frac{\partial h_{j}}{\partial h_{i \neq j}}=-\alpha \frac{h_{j}}{H} \frac{1}{N}$, we observe that the second term disappears when we ignore spill-overs $(\alpha=0)$. See Appendix 5 for more details. 
all cross price derivatives of demand functions will have the same functional form, different from own price derivative, and all cross knowledge derivatives of pricing functions will have the same functional form, different from own knowledge derivative. In addition, in the symmetric equilibrium analysed below, these cross derivatives of demand and pricing functions will have the same value. Therefore, we may consider only two subindexes: $i$ and $j$ as referring to own and cross effects. More formally, $\mathbf{h}_{-i}=\left\{h_{l}\right\}_{l \neq i}$ and $\mathbf{p}_{-i}=\left\{p_{l}\right\}_{l \neq i}$. Thus, index $j$ simply refers to any other firm except $i$. This allows us to rewrite the condition for symmetric equilibria as:

$$
\begin{array}{ll}
h_{i} \quad\left[\frac{\partial x_{i}(\mathbf{p})}{\partial p_{i}} \frac{\partial p_{i}(\mathbf{h})}{\partial h_{i}}+(N-1) \frac{\partial x_{i}(\mathbf{p})}{\partial p_{j}} \frac{\partial p_{j}(\mathbf{h})}{\partial h_{i}}\right]+ \\
+h_{i} x_{i}(\mathbf{p})\left(\frac{\partial p_{i}(\mathbf{h})}{\partial h_{i}}+\frac{w}{h_{i}^{2}}\right)+ \\
+\quad(1-\alpha) w L_{I_{i}}=\frac{w}{\gamma} \frac{h_{i}^{\alpha}}{H^{\alpha}}\left[-\frac{\dot{w}_{t}}{w_{t}}+(1-\alpha) \frac{\dot{h}_{i, t}}{h_{i, t}}+\alpha \frac{\dot{H}_{t}}{H_{t}}+r_{t}\right],
\end{array}
$$

where $\frac{\partial x_{i}(\mathbf{p})}{\partial p_{j}}$ and $\frac{\partial p_{j}(\mathbf{h})}{\partial h_{i}}$ refer to partial derivatives with respect to price or knowledge base of any other firm, evaluated at symmetry. Simplifying and using the condition for optimal pricing from the second stage in (17), we get:

$$
\begin{gathered}
h_{i}\left[(N-1) \epsilon\left(x_{i} ; p_{j} \mid p_{-j}\right) \frac{x_{i}}{p_{j}} \frac{\partial p_{j}\left(h_{j}, \mathbf{h}_{-j}\right)}{\partial h_{i}}\left(p_{i}-\frac{w}{h_{i}}\right)+\frac{w}{h_{i}^{2}}\right]+ \\
+\quad(1-\alpha) w L_{I_{i}}=\frac{w}{\gamma} \frac{h_{i}^{\alpha}}{H^{\alpha}}\left[-\frac{\dot{w}_{t}}{w_{t}}+(1-\alpha) \frac{\dot{h}_{i, t}}{h_{i, t}}+\alpha \frac{\dot{H}_{t}}{H_{t}}+r_{t}\right] .
\end{gathered}
$$

We need to evaluate the expressions for the cross price elasticity of demand in the Bertrand case (i.e. holding prices of others constant), $\epsilon\left(x_{i} ; p_{j} \mid \mathbf{p}_{-j}\right)$, and 
the cross derivative of pricing function with respect to the other's knowledge base, $\frac{\partial p_{j}(\ldots)}{\partial h_{i}} 10$. The elasticity can be readily obtained from $(7)$ as $\frac{\epsilon-1}{N}$, and Appendix 1 demonstrates that from (7) and (17), we may obtain:

$$
\frac{\partial p_{j}\left(h_{j}, \mathbf{h}_{-j}\right)}{\partial h_{i}}=-\frac{w}{h_{i}^{2}} d^{B}, d^{B}=\frac{\epsilon(N-1)+1}{(N-1)\left(\epsilon N^{2}-\epsilon(N-1)-1\right)}>0 .
$$

By substituting these expressions for (34) and then simplifying, we find the following expression a necessary condition for intertemporal optimality of a firm's decision making:

$$
\begin{aligned}
x_{i}(\mathbf{p}) p_{i}(\mathbf{h}) \frac{e^{B}-1}{e^{B}} A^{B}(N, \epsilon)+\ldots \\
+(1-\alpha) w L_{I_{i}}=\frac{w}{\gamma} \frac{h_{i}^{\alpha}}{H^{\alpha}}\left[-\frac{\dot{w}_{t}}{w_{t}}+(1-\alpha) \frac{\dot{h}_{i, t}}{h_{i, t}}+\alpha \frac{\dot{H}_{t}}{H_{t}}+r_{t}\right], \\
A^{B}(N, \epsilon)=\frac{(N-1)(1-\epsilon) d^{B}+N e^{B}}{e^{B} N} .
\end{aligned}
$$

The behaviour of term $A^{B}$, which is a function of a number of firms existing in equilibrium and a degree of substitutability of $\mathrm{X}$ varieties, will later prove very useful when comparing market equilibria to socially optimal outcomes. Finally, noting that $L_{X_{i}}=\frac{x_{i}}{h_{i}}$ from (10), and making use of (17), we obtain:

$$
L_{X_{i}} A^{B}(N, \epsilon)+(1-\alpha) L_{I_{i}}=\frac{1}{\gamma} \frac{h_{i}^{\alpha}}{H^{\alpha}}\left[-\frac{\dot{w}_{t}}{w_{t}}+(1-\alpha) \frac{\dot{h}_{i, t}}{h_{i, t}}+\alpha \frac{\dot{H}_{t}}{H_{t}}+r_{t}\right]
$$

Cournot competition The necessary condition for describing the firm's indifference to investing in its knowledge base or production for Cournot compe-

\footnotetext{
${ }^{10}$ If we extended the strategic effects to comprise the spill-over channel, the cross derivative $(N-1) \frac{\partial p_{j}(\ldots)}{\partial h_{i}}$ would be replaced by $\frac{\partial p_{j}(\ldots)}{\partial h_{i}}\left[1-(N-2) \frac{\alpha}{N} \frac{h_{j}}{H}\right]-\frac{\alpha}{N} \frac{h_{j}}{H} \frac{\partial p_{j}}{\partial h_{j}}$.
} 
tition takes the following form:

$$
\begin{aligned}
& \frac{\partial H_{i}^{C}}{\partial h_{i}}= \\
& e^{-\int_{t}^{s} r_{\tau} d \tau}\left[\frac{\partial x_{i}\left(h_{\left.i, \mathbf{h}_{-i}\right)}\right.}{\partial h_{i}}+x_{i}(\mathbf{h}) \nabla p_{i}(\mathbf{x})\left(\frac{\frac{\partial x_{i}\left(h_{i,} \mathbf{h}_{-i}\right)}{\partial h_{i}}}{\frac{\partial \mathbf{x}_{-i}\left(h_{i,} \mathbf{h}_{-i}\right)}{\partial h_{i}}}\right)+x_{i}(\mathbf{h}) \frac{w}{h_{i}^{2}}\right]+ \\
+\quad & \lambda_{i}(1-\alpha) \gamma\left(\frac{H}{h_{i}}\right)^{\alpha} L_{I_{i}}=-\dot{\lambda}_{i} .
\end{aligned}
$$

Substituting (31) and (32), we obtain:

$$
\begin{aligned}
& h_{i}\left[\frac{\partial x_{i}\left(h_{\left.i, \mathbf{h}_{-i}\right)} \partial h_{i}\right.}{2}+x_{i}(\mathbf{h}) \nabla p_{i}(\mathbf{x})\left(\frac{\partial x_{i}\left(h_{\left.i, \mathbf{h}_{-i}\right)}\right.}{\left.\partial h_{i} \mathbf{h}_{-i}\right)}\right)+x_{i}(\mathbf{h}) \frac{w}{\partial h_{i}}\right]+ \\
+\quad(1-\alpha) w L_{I_{i}}=\frac{w}{\gamma} & \frac{h_{i}^{\alpha}}{H^{\alpha}}\left[-\frac{\dot{w}_{t}}{w_{t}}+(1-\alpha) \frac{\dot{h}_{i, t}}{h_{i, t}}+\alpha \frac{\dot{H}_{t}}{H_{t}}+r_{t}\right] .
\end{aligned}
$$

Again, conventional treatment disregards the role research decisions have on the production behaviour of rivals. Hence, the term $\frac{\partial \mathbf{x}_{-i}\left(h_{i}, \mathbf{h}_{-i}\right)}{\partial h_{i}}$ is missing in the standard approach (see K-S).

Using the functional symmetry of prices with respect to the rival firms' levels of production, and production with respect to the rivals' stock of knowledge we may rewrite this condition for a symmetric equilibrium as follows:

$$
\begin{gathered}
h_{i}\left[\begin{array}{c}
\frac{\partial x_{i}\left(h_{i} \mathbf{h}_{-i}\right)}{\partial h_{i}}+x_{i}(\mathbf{h}) \frac{w}{h_{i}^{2}}+\ldots \\
+x_{i}(\mathbf{h})\left(\frac{\partial p_{i}(\mathbf{x})}{\partial x_{i}} \frac{\partial x_{i}(\mathbf{h})}{\partial h_{i}}+(N-1) \frac{\partial p_{i}\left(x_{i}, \mathbf{x}_{-i}\right)}{\partial x_{j}} \frac{\partial x_{j}\left(h_{j,} \mathbf{h}_{-j}\right)}{\partial h_{i}}\right)
\end{array}\right]+ \\
+\quad(1-\alpha) w L_{I_{i}}=\frac{w}{\gamma} \frac{h_{i}^{\alpha}}{H^{\alpha}}\left[-\frac{\dot{w}_{t}}{w_{t}}+(1-\alpha) \frac{\dot{h}_{i, t}}{h_{i, t}}+\alpha \frac{\dot{H}_{t}}{H_{t}}+r_{t}\right]
\end{gathered}
$$

where again we employed notational convention of index $j$ denoting any other firm except $i$. By invoking the pricing condition from the second stage (17) and 
simplifying, we receive:

$$
\begin{gathered}
h_{i} x_{i}(\mathbf{h})\left((N-1) \epsilon\left(p_{i} ; x_{j} \mid \mathbf{x}_{-j}\right) \frac{p_{i}(\mathbf{x})}{x_{j}} \frac{\partial x_{j}(\mathbf{h})}{\partial h_{i}}+\frac{w}{h_{i}^{2}}\right)+ \\
+\quad(1-\alpha) w L_{I_{i}}=\frac{w}{\gamma} \frac{h_{i}^{\alpha}}{H^{\alpha}}\left[-\frac{\dot{w}_{t}}{w_{t}}+(1-\alpha) \frac{\dot{h}_{i, t}}{h_{i, t}}+\alpha \frac{\dot{H}_{t}}{H_{t}}+r_{t}\right] .
\end{gathered}
$$

We need to evaluate the expressions for the cross price elasticity of demand in the Cournot case (i.e. holding quantities of others constant), $\epsilon\left(x_{i} ; p_{j} \mid \mathbf{x}_{-j}\right)$, and the cross derivative of the quantity function with respect to another's knowledge base, $\frac{\partial x_{j}(\mathbf{h})}{\partial h_{i}}$. The elasticity can be readily obtained from (8) as $\frac{1-\epsilon}{\epsilon N}$, and Appendix 1 demonstrates that from (8) and (17), we may obtain:

$$
\frac{\partial x_{j}(\ldots)}{\partial h_{i}}=-\frac{w}{h_{i}^{2}} \frac{x_{i}}{p_{i}} d^{C}, d^{C}=\frac{\epsilon(N-2)}{(N-1)(\epsilon+N-2)} \geq 0 .
$$

Upon using these expressions in (37) and simplifying, we obtain the following expression as a necessary condition (in a symmetric equilibrium) for intertemporal optimality of a firm's decision making when playing Cournot in the second stage:

$$
\begin{gathered}
x_{i} p_{i} \frac{e^{C}-1}{e^{C}} A^{C}(N, \epsilon)+(1-\alpha) w L_{I_{i}}= \\
=\frac{w}{\gamma} \frac{h_{i}^{\alpha}}{H^{\alpha}}\left[-\frac{\dot{w}_{t}}{w_{t}}+(1-\alpha) \frac{\dot{h}_{i, t}}{h_{i, t}}+\alpha \frac{\dot{H}_{t}}{H_{t}}+r_{t}\right] \\
A^{C}(N, \epsilon)=\frac{(\epsilon-1)(N-1) d^{C}+\epsilon N}{\epsilon N} .
\end{gathered}
$$

Finally, noting that $L_{X_{i}}=\frac{x_{i}}{h_{i}}$ from (10) and making use of (17), we obtain: 


$$
L_{X_{i}} A^{C}(N, \epsilon)+(1-\alpha) L_{I_{i}}=\frac{1}{\gamma} \frac{h_{i}^{\alpha}}{H^{\alpha}}\left[-\frac{\dot{w}_{t}}{w_{t}}+(1-\alpha) \frac{\dot{h}_{i, t}}{h_{i, t}}+\alpha \frac{\dot{H}_{t}}{H_{t}}+r_{t}\right]
$$

We can observe that the intertemporal condition for a firm's behaviour, when Cournot is played at the second stage (39), has the same form as the condition when Bertrand is played (35) with term $A^{B}$ replaced by $A^{C}$. These terms are different functions of the elasticity of substitution $\epsilon$ and the number of firms $N$. Hence, when the number of firms is kept exogenously small (when free entry is prohibited), such a formulation of intertemporal conditions makes the effects of different modes of competition on equilibrium particularly easy to analyse. The terms can be understood as measures of strategic interactions linking the decisions about $R \& D$ with those about pricing and production. Note in particular that when firms ignore the effects of their research investment on the others' pricing and quantity decisions (the terms $\frac{\partial p_{j}(\ldots)}{\partial h_{i}}$, and $\frac{\partial x_{j}(\ldots)}{\partial h_{i}}$ above are set to zero), the terms $d^{C}$ and $d^{B}$ are both zero, and $A^{B}$ and $A^{C}$ are equal to one. In this case, these measures of strategic interactions disappear from the above conditions and the mode of competition is not important. Similarly, if the number of firms $N$ reaches infinity, both strategic terms converge to unity, because strategic interactions become unimportant. This is a standard result (van de Klundert and Smulders, 1997). Note also that the effects of strategic interactions we consider here do not hinge on the existence of knowledge spillovers.

Having characterised the paths of a firm's optimal choices, we may also characterise the firm's intertemporal profits, i.e. the value of a firm in (12) in a 
symmetric equilibrium by using (24) or (29):

$$
\begin{aligned}
V_{i, t} & =\int_{t}^{\infty} e^{-\int_{t}^{s} r_{\tau} d \tau}\left[\pi_{i, s}-w_{s} L_{I i, s}\right] d s \\
& =\int_{t}^{\infty} e^{-\int_{t}^{s} r_{\tau} d \tau}\left[\frac{E_{s} \sigma}{N_{s} e}-w_{s} f-w_{s} L_{I i, s}\right] d s
\end{aligned}
$$

This completes the description of the behaviour of $\mathrm{X}$ sector firms and we move next to characterisation of equilibria.

\section{Market Equilibrium}

For analytical purposes, we are only interested in symmetric equilibria among $\mathrm{X}$ sector firms. Hence, all hi-tech firms are identical in equilibrium, producing the same quantities at identical prices and investing the same resources in the development of their knowledge bases. This makes an average industry level of knowledge, $H$, identical to a representative firm's knowledge, $h$, significantly simplifying the analysis.

Market equilibrium is the combination of all allocations and prices in the model economy such that the behaviour of all agents is optimal and all markets clear. The clearance of goods markets has already been ensured by the optimal behaviour of firms. The clearance of bond markets implies that household savings are used to finance R\&D investment:

$$
\dot{S}=w \sum_{i=1}^{N} L_{I, i} .
$$

The intertemporal budget constraint in (3) and (4) can be, after substituting for the firm's profits (22 and 29) and labour demands (46, 9 and 6), manipulated 
to yield in symmetry:

$$
\begin{aligned}
E_{C} & =w N\left[L_{X}+f\right]+w L_{Y}+N \pi . \\
E_{C} & =w N L_{X}+w L_{Y}+\frac{E \sigma}{e} \\
E_{C} & =\frac{E w}{e-\sigma}\left(N L_{X}+L_{Y}\right) \\
E_{C} & =\frac{N L_{X} e w}{\sigma(e-1)} .
\end{aligned}
$$

The only other market that matters here is the labour market in which all the workforce must equal the exogenously given supply of labour, $L$ :

$$
L=L_{Y}+N\left(L_{X}+f\right)+N L_{I} .
$$

Using (45) in labour demand in sector $Y$ given by equations (9) and (6) we obtain the following expression regarding the optimal allocation of labour between production and research:

$$
N L_{X} \frac{e-\sigma}{\sigma(e-1)}+N L_{I}=L-N f .
$$

The symmetric equilibrium with a fixed number of $\mathrm{X}$ varieties (henceforth denoted as a constrained equilibrium) can then be characterised by the following system of equations:

$$
\begin{aligned}
\frac{\dot{E}_{C, t}}{E_{t}} & =r_{t}-\theta, \\
\frac{\dot{h}_{t}}{h_{t}} & =\gamma L_{I_{i, t}},
\end{aligned}
$$




$$
\begin{aligned}
L_{X, t} \frac{\dot{h}_{t}}{h_{t}} A^{B}(N, \epsilon) & =L_{I, t}\left[-\frac{\dot{w}_{t}}{w_{t}}+\alpha \frac{\dot{h}_{i, t}}{h_{i, t}}+r_{t}\right], \\
E_{C, t} & =\frac{N_{t} L_{X, t} e w_{t}}{\sigma(e-1)}, \\
N_{t} L_{X, t} \frac{e-\sigma}{\sigma(e-1)}+N_{t} L_{I, t} & =L-N_{t} f .
\end{aligned}
$$

The system defines the evolution of allocations $E, h, L_{I}, L_{X}$, and prices $r, w$, for the given parameters and number of $\mathrm{X}$ firms. The first equation describes the consumer's intertemporal behaviour as in (5). The second is a symmetric equilibrium version of the technological constraint in (11). The third describes the optimal behaviour of $\mathrm{X}$ sector firms. It is derived from (35) (or 39) by imposing symmetry and first substituting $L_{I_{i}}$ by (48) and then $\frac{L_{I_{i}}}{h_{i} / h}$ by $\frac{1}{\gamma}$ in the resulting expression. The last two are products of the asset market and labour market clearing conditions and were already derived earlier.

In a free entry equilibrium, new $\mathrm{X}$ firms enter as long as the intratemporal profits are positive, so the number of firms can be pinned down by the following condition:

$$
V=0 \text {. }
$$

$V$ may be determined from (41) and (45) as:

$$
V=\int_{t}^{\infty} e^{-\int_{t}^{s} r_{\tau} d \tau}\left[\left(\frac{L_{x_{i}, s}}{(e-1)}-f-L_{I i, s}\right)\right] d s
$$

The value of a firm on a balanced growth path (BGP) decreases in the number of competing $\mathrm{X}$ sector firms, other things being equal, because the partial derivative of $\frac{\partial e^{B, C}}{\partial N}>0$ (see 20). If the number is determined exogenously, the 
value of an incumbent firm will be non-negative.

Because only relative prices can be solved in the equilibrium, we employ the normalisation suggested above, namely $w=P_{Y}=1$, and express all variables in terms of traditional goods. As usual, we will concentrate on analysing the system at a balanced growth path (BGP). There are no transitional paths, and the system, once off the BGP, immediately jumps back. We are looking for balanced growth paths with knowledge accumulating at a constant rate $g$. This implies that a constant fraction of labour resources is devoted to knowledge accumulation, and hence also to the production of X goods. Then, total expenditure will be constant at the BGP, which can only be if the interest rates are also constant and equal to the subjective discount rate. Hence, the balanced growth (constrained) equilibrium is described by the following modification to the above differential system:

$$
\begin{aligned}
r_{t} & =\theta \\
g & =\frac{\dot{h}_{i}}{h}=\gamma L_{I_{i}}, \\
L_{X_{i}} g A^{B}(N, \epsilon) & =L_{I}\left(\alpha g+r_{t}\right), \\
E & =\frac{N L_{X} e w}{\sigma(e-1)}, \\
N L_{X} \frac{e-\sigma}{\sigma(e-1)}+N L_{I} & =L-N f .
\end{aligned}
$$

If we allowed a free entry, this system would be accompanied by the freeentry condition (52) which on the BGP implies: 


$$
\frac{L_{x_{i}, s}}{(e-1)}-f-L_{I i}=0 .
$$

The system can be easily solved for the balanced rate of growth. Solving the system of (57) and (55) for $L_{I}$ and $L_{X}$ and substituting the resulting expression for $L_{I}$ in (54) we obtain the following expression for the balanced rate of growth as a function of parameters and the number of $\mathrm{X}$ sector firms:

$$
g(N, e)=\frac{\gamma \sigma(e-1)\left(\frac{L}{N}-f\right) A-\theta(e-\sigma)}{\alpha(e-\sigma)+A \sigma(e-1)},
$$

where $e$ and $A$ take the form according to the mode of competition. Examining the expressions for $A$ offers direct insight into the effects of competition on growth for a given level of concentration (defined as $1 / N$ ). In a free entry equilibrium (when the level of concentration is endogenous), the reduced form expression for $g$ and $N$ are difficult to obtain. Nevertheless, it would be beneficial to complement condition (59) with a condition determining the number of firms in a free entry equilibrium. Substituting the expression obtained above for $L_{X}$ in equation (58) and using (54), we find:

$$
g(N, e)=\gamma\left(\frac{L \sigma}{N e}-f\right) .
$$

The system of (59) and (60) determines the equilibrium values of growth and the number of hi-tech firms along the BGP as functions of the model's parameters. 


\section{The effects of strategic interactions}

The constrained rate of growth in condition (59) differs from the conventional result by featuring strategic terms $A^{C}$ or $A^{B}$. Specifically, if we were to follow the K-S analysis to the letter, we would have found the equivalent condition to be:

$$
g(N, e)=\frac{\gamma \sigma(e-1)\left(\frac{L}{N}-f\right)-\theta(e-\sigma)}{\alpha(e-\sigma)+\sigma(e-1)} .
$$

Regarding terms $A^{C}$ and $A^{B}$, we have already said informally that their values indicate a measure of strategic interactions among $\mathrm{X}$ sector firms, approaching unity as the number of firms goes to infinity. The appendix 2 provides further details about the behaviour of these expressions on the domain of plausible parameter values. The main difference between the two is that while $A^{B}$ approaches unity from below, $A^{C}$ does it from above. The second difference is that while the partial derivative of $A^{B}$ with respect to $N$ is monotonic, $A^{C}$ is generally non-monotonic and reaches its maximum at values of $N$ close to 3 . Third, at the limit value $N=2, A^{B}$ approaches exactly $2 / 3$ as $\epsilon$ reaches infinity while $A^{C}$ is unity. This finding implies that when there are only two firms playing Cournot at the second stage, there cannot be any strategic interactions among them in the first stage. These only become important at lower levels of concentration. The reason for this peculiar result is that when there are only two firms playing Cournot at the second stage, an increase in one's production has no effect on the marginal revenues of the other (see Appendix 2). These observations are best manifested in Figure 1 depicting the expressions in $\epsilon, N$ 
space.

To better understand the mechanisms operating in these strategic variables, we rewrite them in terms of various elasticities considered throughout the paper. It can be shown that for Bertrand competition ${ }^{11}$ :

$$
A^{B}=1-(N-1) \frac{\epsilon\left(x_{i} ; p_{j} \mid p_{-j}\right) \epsilon\left(p_{j} ; h_{i}\right)}{1-e^{B}} .
$$

The variable differs from 1 by the expression $(N-1) \frac{\epsilon\left(x_{i} ; p_{j} \mid p_{-j}\right) \epsilon\left(p_{j} \mid h_{i}\right)}{1-e^{B}}>0$. Consider now the elasticities in the numerator of this expression. They show the anticipated percentage effect of the $R \& D$ investment of a representative firm $i$ on the contraction of demand for its products through the pricing reaction of a rival firm $j$. Elasticity $\epsilon\left(p_{j} ; h_{i}\right)$ describes the channel through which the R\&D investment alters optimal pricing by its rivals. Elasticity $\epsilon\left(x_{i} ; p_{j} \mid p_{-j}\right)$, on the other hand, shows the response of consumer demand to a pricing reaction of the rival firm, holding the prices of others constant. It is the ordinary cross price elasticity of demand for Bertrand competition. As there are $N-1$ identical rival firms in the symmetric equilibrium, the total effect on the demand for the goods of the investing firm results after multiplying by this number. The fraction part of the expression has this total effect of $R \& D$ investment on the firm's own demand expressed relative to the percentage demand effect of the own price decision (its excess over 1).

In Cournot competition, $A^{C}$ can be rewritten as ${ }^{12}$ :

\footnotetext{
${ }^{11}$ From condition (34) and the subsequent exposition, we observe that $A^{B}=1+(N-$ 1) $\frac{\epsilon\left(x_{i} ; p_{j} \mid p_{-j}\right)}{e^{B} c^{\prime}\left(h_{i}\right)} \frac{\partial p_{j}(.)}{\partial h_{i}}=1+(N-1) \frac{\epsilon\left(x_{i} ; p_{j} \mid p_{-j}\right)}{e^{B} c^{\prime}\left(h_{i}\right)} \frac{p_{j}}{h_{i}} \epsilon\left(p_{j} ; h_{i}\right)$.

Recalling further that $\frac{p_{j}}{c^{\prime}\left(h_{i}\right) h_{i}}=\frac{e^{B}}{e^{B}-1}$ gives us the result.

${ }^{12}$ Note that from (37) and the subsequent exposition we can write $A^{C}$ as $A^{C}=1-(N-$
} 


$$
A^{C}=1+(N-1) \frac{e^{C} \epsilon\left(p_{i} ; x_{j} \mid x_{-j}\right) \epsilon\left(x_{j} ; h_{i}\right)}{1-e^{C}} .
$$

Although seemingly different, the expression by which $A^{C}$ exceeds $1,(N-$ 1) $\frac{e^{C} \epsilon\left(p_{i} ; x_{j} \mid x_{-j}\right) \epsilon\left(x_{j} \mid h_{i}\right)}{1-e^{C}}>0$, is interpreted identically to the equivalent expression in the Bertrand case. Elasticity $\epsilon\left(x_{j} \mid h_{i}\right)$ comprises the link, in percentage terms, through which a firm's own R\&D investment affects the production decision of a representative rival. Elasticities $\epsilon\left(p_{i} ; x_{j} \mid x_{-j}\right) e^{C}$ then translate this effect into the impact on consumer demand for the production of the investing firm. We note too that $e^{C} \equiv-\epsilon\left(x_{i} ; p_{i} \mid x_{-i}\right)$. As before, the total effect is normalised with respect to the percentage demand effect of the firm's own price decision (its excess over 1 ).

In both cases, therefore, the strategic term $A$ measures the effect of $\mathrm{R} \& \mathrm{D}$ expenditure on the consumer demand of the investing firm, which can be anticipated from the reaction of its rivals. In Appendix 5, we demonstrate that so defined strategic expressions and their position in the definition of the equilibrium remain intact, even when allowing for the interactions to pass through the knowledge spill-over channel (although, if expressed in primitive parameters, the expressions would differ substantially). This is an important result, because it permits us to think about these expressions as conjectural variables reflecting the 'anticipated' strategic effects of R\&D investment. Such an interpretation makes way for their easier empirical calibration.

1) $\frac{\epsilon\left(p_{i} ; x_{j} \mid x_{-j}\right)}{c^{\prime}\left(h_{i}\right)} \frac{p_{i}}{x_{j}} \frac{\partial x_{j}(.)}{\partial h_{i}}=1-(N-1) \frac{\epsilon\left(p_{i} ; x_{j} \mid x_{-j}\right)}{c^{\prime}\left(h_{i}\right)} \frac{p_{i}}{h_{i}} \epsilon\left(x_{j} ; h_{i}\right)$. Recalling further that $\frac{p_{i}}{c^{\prime}\left(h_{i}\right) h_{i}}=$ $\frac{e^{C}}{e^{C}-1}$ gives us the result. 


\section{The effects of competition and social optimal- ity}

In order to grasp the implications of different modes of competition for balanced growth rates, we need to analyse the relationship between terms $e^{B}$ and $e^{C}$, and between $A^{C}$ and $A^{B}$. As for the elasticities, the expressions in (20) clearly show that in a symmetric equilibrium $e^{B}>e^{C}$. In other words, because the perceived price elasticity of demand is higher, mark-ups over marginal costs must be lower under the tougher Bertrand competition. We may then establish the implications of competition toughness for growth by partially differentiating the expression in (59):

$$
\frac{\partial g(N, e, A)}{\partial e}=\frac{A \sigma(1-\sigma)}{[\alpha(e-\sigma)+A \sigma(e-1)]^{2}}\left[\gamma\left(\frac{L}{N}-f\right) \alpha+\theta\right] \geq 0 .
$$

The higher price elasticity perceived under Bertrand competition leads to higher balanced rates of growth for any given level of concentration and strategic interaction terms ${ }^{13}$. This is a standard result, analysed, for instance, by van de Klundert and Smulders: Intuitively, lower prices of hi-tech goods under Bertrand competition make them relatively cheaper than traditional goods, and so the market and profits of hi-tech firms are larger. This increases incentives for research investment. However, the two modes of competition differ not only in pricing rules, but also in the impacts of strategic interactions, as exemplified by terms $A^{C}$ and $A^{B}$. These were missing in original van de Klundert and

\footnotetext{
${ }^{13}$ Note that the gradient of $A^{C}-A^{B}$ also contains the difference between $e^{C}$ and $e^{B}$, because in general, $A$ is a function of $e$. Thus, the partial derivative above, holding $A$ constant, underestimates the elasticity effect because it ignores the channel that works through strategic interactions (see below).
} 
Smulders' original exposition.

If we look solely to the implications of the strategic terms, condition (59) suggests that when $A^{B} \leq 1$ and $A^{C} \geq 1$, the balanced growth rates under Bertrand competition will probably fall short of Cournot rates for any given degree of concentration, $N$, and perceived price elasticity, $e$. This intuition is supported by condition (55), which gives the ratio of resources devoted to research and output implied by the intertemporal optimisation of firms: for any given combination of parameters and growth rate, the relative resources devoted to research will be higher for firms engaged in Cournot, and not Bertrand, competition. To support this intuition, we differentiate the expression for constrained balanced growth (59) with respect to the degree of strategic interactions $A$, holding the level of concentration and the perceived price elasticity constant ${ }^{14}$ :

$$
\frac{\partial g(N, e, A)}{\partial A}=\frac{\sigma(e-1)(e-\sigma)}{[\alpha(e-\sigma)+A \sigma(e-1)]^{2}}\left[\gamma\left(\frac{L}{N}-f\right) \alpha+\theta\right] \geq 0 .
$$

Indeed, because $A^{C} \geq A^{B}$, the constrained balanced growth under Cournot competition is higher than under Bertrand, for any given level of concentration (and perceived price elasticity). And, as condition (60) remains unaffected by the mode of competition, this result implies that when ignoring different price

\footnotetext{
${ }^{14}$ It may seem inconsistent to differentiate the growth rate with respect to $A$, while keeping the perceived elasticity constant, when, in fact, it changes across the mode of competitions for the same parameter values. This is certainly true for the well-specified strategic game space considered here. But empirically, it helps to understand the perceived price elasticity $e$ as a measure of competition toughness, as for instance in Sutton (1991). Under such circumstances, $e$ may be treated as the primitive parameter of the remaining system, even though it does not result from a properly defined intratemporal game. The perceived elasticity may function as an ad hoc conjecture, just as the 'conjectural variation' parameter in the IO literature (see e.g. Brander, 1995). If we likewise treat $A$ as a primitive conjecture on strategic effects, the derivative of the growth rate with respect to $A$, holding $e$ constant, would be meaningful. In addition, the derivative below examines the effect of including a strategic term in the conventioanal functional form for the constrained growth rate.
} 
elasticities in a free entry equilibrium, Cournot growth rate is higher and the number of hi-tech firms lower than under Bertrand.

This result can be understood as follows. Because the choice variables (prices) under Bertrand are strategic substitutes, while under Cournot they (quantities) are complements, a firm's rivals react to its lower price by following suit. The implication is that by investing in its own $R \& D$, the firm is able to charge lower prices. This triggers an optimal response from its rivals, who adjust their prices downward to eliminate the price disadvantage. When the investing firm takes this into account, its perceived profits from production decrease, making the incentive to invest in R\&D lower than under Cournot. Under Cournot, by contrast, rival firms react to a firm's increased production by decreasing their own production. Because investment in research enables the firm to increase production, the rival firms' optimal response is to decrease their production, which in turn makes the profits of the investing firm comparatively higher. This enhances the benefits of research and leads to a higher rate of knowledge accumulation. However, note that reallocation of more labour resources to R\&D under Cournot competition actually decreases the life-time profits of hi-tech firms (conditions 52 and 58) as compared to the Bertrand case, so only fewer firms can be supported in a free entry market equilibrium.

The effects of competition toughness and strategic interaction, therefore, have contrary implications for constrained growth rates under the two modes of competition. Which of them dominates is difficult to tell in general, but we can make few observations. For instance, both effects disappear as $N \rightarrow \infty$. Note 
also that the effect of tougher competition relies on the presence of traditional goods. Ignoring the traditional sector (by setting $\sigma=1$ ) removes the relative price distortion, so only strategic interactions remain to distinguish between the two modes.

In this context, Cournot constrained growth rates exceed those of Bertrand. As we show in Appendix 3 using numerical simulations, the share of hi-tech goods in consumption is a critical parameter determining which mode of competition brings about a higher balanced growth rate. We demonstrate, that in an approximation of the problem, for several levels of concentration there exists a critical level of this share $(\tilde{\sigma})$ for any degree of substitutability among hi-tech goods $(\epsilon)$ above which Cournot growth rates dominate those of Bertrand. This critical value rises with higher substitutability, making higher Bertrand growth rates more likely (Figure 2). On the other hand, it falls with a greater number of competing firms, making higher Bertrand growth rates more unlikely. From our approximation of the problem, then, it appears that higher constrained growth rates may be observed under Bertrand competition only at low levels of concentration (close to duopoly) when the share of hi-tech goods in consumption is also relatively low; a constellation that may prove rare in practice.

Although the size of the approximating error remains undetermined, we can firmly concluded that if the share of goods, for whose production learning is important, is high enough, the more relaxed Cournot competition yields a higher balanced growth rate. We could not obtain these kinds of results in a standard treatment, which ignores the effects of strategic interactions linking 
decisions about $\mathrm{R} \& \mathrm{D}$ with those of pricing and production.

\subsection{Social optimality}

A detailed solution to the problem of an idealised social planner looking for the growth rate and the number of firms which would maximise households' utility can be found in Smulders and van de Klundert (1997). There, the socially optimal balanced rate of growth for a given number of hi-tech firms (i.e. socially optimal constrained rate of growth) is given as:

$$
g(N, e)=\frac{\gamma \sigma\left(\frac{L}{N}-f\right)-\theta}{\sigma},
$$

and the optimal number of firms is determined by:

$$
g(N, e)=\gamma\left(\frac{L \sigma}{N(\epsilon-1+\sigma)}-f\right) .
$$

As the authors note, market equilibrium will in general differ from the social optimal outcome because of three types of imperfections. First, because of knowledge spill-overs in investing $(\alpha>0)$, firms do not appropriate all the benefits of R\&D investment, causing it to be suboptimally low (ceteris paribus). Second, because of imperfect competition in the $\mathrm{X}$ sector, the relative price of hi-tech versus traditional goods is higher than the marginal rate of technical substitution, again leading to lower production of hi-tech goods and a lower rate of innovation. It is easily demonstrated that by removing these distortions through the elimination of spill-overs $(\alpha=0)$, elimination or taxation of the traditional sector ( $\sigma=1$ or tax to correct for the relative price) and ignorance of 
the strategic terms, condition (59) becomes identical to (65). Then the market and social rates of growth, conditional on a given rate of concentration, coincide in the absence of strategic interactions. The third type of distortion involves the optimal number of firms in a free-entry equilibrium. Because firms base their pricing on perceived demand elasticities $(e)$ which are different (for small values of $N$ ) from consumers' demand elasticities $(\epsilon)$, the firms' profits are too large and too many firms co-exist in the equilibrium. In addition to the three outlined by K-S, there is also a forth type of imperfection caused by strategic interactions when the number of firms is small, and depending on the mode of competition. Under Cournot, strategic complementariness of production (as the choice variable of firms) motivates further research investment, making it too high from a social point of view. Under Bertrand, by contrast, the strategic substitutability of prices decreases the benefits of research, leading to a suboptimally low level of investment.

As we are primarily interested in the effects of competition on market growth rates, we dismiss the other imperfections. First, we remove the traditional sector $(\sigma=1)$, and second, we only work with a fixed number of X-sector firms, thus avoiding the second and third types of imperfections. The (constrained) market growth rate is then given by:

$$
g(N, e)=\frac{\gamma\left(\frac{L}{N}-f\right) A-\theta}{\alpha+A},
$$

while the socially optimal rate becomes: 


$$
g^{S P}(N, e)=\gamma\left(\frac{L}{N}-f\right)-\theta .
$$

Note that when the number of firms goes to infinity (strategic interactions lose importance, $A=1$ ) and there are no spill-overs (externality disappears, $\alpha=$ 0 ), the market and social rates of growth coincide. It follows that if we ignore only strategic interactions $(A=1)$, then the market growth rate will always fall short of the social rate and the spill-over imperfection will never be corrected without introducing taxes or subsidies. This is the same result obtained by van de Klundert and Smulders, who ignored the strategic interactions when investing in R\&D. We find, however, that incorporating these interactions may trigger their result either way.

In order to support intuition first note that with no spill-overs the market growth rate becomes:

$$
g(N, e)=\gamma\left(\frac{L}{N}-f\right)-\frac{\theta}{A}
$$

and will either exceed the social rate (Cournot) or fall short (Bertrand). That Cournot delivers too high growth rate is a consequence of strategic interactions linking decisions about $\mathrm{R} \& \mathrm{D}$ to production, which do not rely on the spill-over channel. These results thus parallel the discussion in strategic trade literature (Brander, 1995).

With spill-overs and interactions back in place, the two rates may coincide under the following condition for $A$ : 


$$
A=1-\alpha+\frac{\alpha \gamma\left(\frac{L}{N}-f\right)}{\theta}=1+\alpha \frac{g^{S P}(N)}{\theta} \geq 1
$$

Because $A^{B} \leq 1$, it follows that Bertrand balanced growth rates will always fall short of socially optimal (constrained) growth rates, for any degree of concentration. This is easily understood, since in this case both the spill-over externality and strategic substitutability of prices decrease research investment. In addition, the rate of knowledge accumulation will be lower than what is implied by van de Klundert and Smulders. But for Cournot competition, $A^{C} \geq 1$ and hence the socially (constrained) optimal rate of growth may in principle be attained. The reason is that this time, spill-over externality works against the strategic complementariness of production in the final effect on knowledge accumulation. In this vein, strategic interactions may act as a device that removes the imperfection caused by the inappropriability of research spill-overs. This effect could not be obtained in the original Smuldert and van de Klundert treatment. Formally, the condition for $N$ under which the market and social constrained rates of growth coincide is:

$$
\frac{(\epsilon-1)(N-2)}{N(\epsilon+N-2)}=\alpha \frac{g^{S P}(N)}{\theta} .
$$

This condition gives a cubic equation in $N$ which is non-trivial to analyse explicitly. Nevertheless, we show in Appendix 4 that when restricting ourselves to values of $N$ greater than or equal to 2, we may have only one or no solution satisfying the above condition. Figures in the Appendix suggest where such 
a solution may lie. There we also find that for the coincidence of social and market constrained growth rates on this domain, hi-tech goods must be good enough substitutes. Under this condition, the socially optimal rate of growth may be attained even at fairly high levels of concentration, without imposing taxation on the traditional sector.

\section{Concentration and growth}

The preceding analysis enables us to draw a qualitative conclusion about the relationship between concentration and growth. While ordinarily (ignoring strategic interactions), one would expect balanced growth rates to fall with lower concentration because lower market power reduces profits from innovation, this may not be the case when the number of firms is small and the strategic interactions among them are important. The reason is that different levels of concentration alter the growth implications of strategic interactions. The total effect of a change in the market growth rate when the number of firms increases may thus be decomposed into two effects: a fall in market power and a change in strategic impact. Put formally:

$$
\frac{\partial g(N)}{\partial N}=\frac{\partial g(N, A)}{\partial N}+\frac{\partial g(N, A)}{\partial A} \frac{\partial A(N)}{\partial N} .
$$

The first term, attributable to a fall in market power, is always negative $^{15}$. The second reflects a change in the growth effect of strategic inter-

\footnotetext{
${ }^{15}$ In fact, this first element comprises two effects: a fall in market power (negative sign) and a decline in the hi-tech sector relative to the traditional one (positive sign). It is easy to see, though, that the former always dominates the latter for any level of concentration. However, this would not need to be the case if productivity in a firm's R\&D sector depended on an industry-wide stock of knowledge capital rather than on an industry average as in the present
} 
actions. We know from (64) that $\frac{\partial g(N, A)}{\partial A}$ is always positive and that the sign of $\frac{\partial A(N)}{\partial N}$ depends on the mode of competition. Because for Bertrand competition $\frac{\partial A^{B}(N)}{\partial N}>0$, the overall effect on growth is ambiguous. Nonetheless, as is shown in Appendix 2, $\frac{\partial A^{B}(N)}{\partial N}$ falls rapidly towards zero, much faster than $\frac{\partial g(N, A)}{\partial N}$. We thus draw a qualitative conclusion that at lower levels of concentration, the market growth rate probably falls with a rising number of firms. The speed of this fall is however slower than predicted under a conventional market power analysis, and at lower levels of concentration this relationship may even break down.

Only marginally more precise conclusions can be made for Cournot competition. We show in Appendix 2 that $\frac{\partial A^{C}(N)}{\partial N}<0$ for $N \geq \tilde{N}=2+\sqrt{2 \epsilon}$. On this interval $\frac{\partial g(N)}{\partial N}<0$, that is, the market growth rate falls with a rising number of firms. But this time, the speed of the fall is faster than predicted under conventional loss of market power considerations. Yet, for $N \in\langle 2,2+\sqrt{2 \epsilon}\rangle$ the partial derivative $\frac{\partial g(N)}{\partial N}$ is positive, and again the total effect is ambiguous. In spite of this, we may still conclude that if the balanced growth rates rise at all with lower concentration, then it can only be at very high concentration levels close to duopoly. This unconventional result arises from the consideration of strategic effects.

case. In such a setting with fewer firms, possibilities of inter-firm learning diminish, impacting negatively on growth. This effect is sometimes called the public knowledge effect. Smulders and van de Klundert (1995) show that this effect is likely to overturn the sign of the first element in expression (71) at high levels of concentration. Hence, the public knowledge effect may also cause a non-monotonic relationship between levels of concentration and growth. However, this effect is absolutely distinct from the effects of strategic impacts shown below, even though they too may lead to non-monotonicity in the relationship between concentration and growth. 


\section{Conclusions}

In this paper, we focused on the implications of strategic interactions among hitech firms on the rates of growth when the number of competing firms is small and firms recognise the effects their investment decisions have on the production behaviour of their rivals. Like other results, we find that a mode of oligopolist competition can substantially affect rates of growth, but we present novel channels for these effects. These channels show that less severe Cournot competition delivers rates of growth higher than socially optimal if knowledge spill-overs are absent, although in their presence, the result depends upon parameterisation and the two rates may coincide. On the other hand, a social optimum can never be achieved under the more severe Bertrand competition, where subsidies remain the only means of restoring social optimality. These findings are parallel to the conclusions in the strategic trade literature. There the standard result is that firms tend to under-invest from the social (cost minimising) perspective in Bertrand and over-invest in Cournot competition (Brander, 1995). Our results demonstrate that conclusions from the strategic trade literature are robust enough if extended into an explicitly dynamic general equilibrium framework. More importantly, though, our results make it evident that questions of optimal policy design related to organisational structure and strategic conduct are tractable in the framework of endogenous growth models in sufficient detail.

As is standard in the literature, we confirmed that modes of competition can have opposite effects on balanced growth rates, depending on whether choice variables in this competition are strategic substitutes (price competition) or 
complements (quantity competition). Unlike standard results that stress the positive effect of tougher Bertrand competition on growth, we find ambiguous results depending upon parameterisation, which seems to favour Cournot competition. In particular, if the consumption share of goods, for whose production learning is important, is high enough, the less severe Cournot competition brings about higher growth rates because the pro-growth effect of strategic interactions dominates the traditional competitiveness effect. These interactions imply that firms engaged in price competition face lower returns from their R\&D investment than do firms engaged in quantity competition, and hence invest less, because they correctly anticipate that rivals will react to their investment by lowering prices. On the other hand, a firm engaged in quantity competition anticipates that its rivals will lower production in response to its investment; this increases the perceived profits from R\&D.

While the relation of growth rates under the two modes of competition is ambiguous, their relation to (constrained) socially optimal rates of growth is not. In particular, we found that Bertrand balanced growth rates will fall short of socially optimal (constrained) growth rates for any degree of concentration. The reason is that, in the case of price competition, both the spill-over externality and strategic substitutability of prices decrease research investment. By contrast, for quantity competition the socially (constrained) optimal rate of growth may in principle be attained. This time, the spill-over externality goes against the strategic complementariness of production in the final effect on knowledge accumulation. In this vein, strategic interactions may serve as a 
device that removes the imperfection caused by the inappropriability of research spill-overs. The directions and sizes of these effects could not be obtained in the original Smulders and van de Klundert treatment. We also showed that in the absence of knowledge spill-overs, Cournot competition will always deliver excessively high growth rates.

Unfortunately, we were not able to say much about the relationship between levels of concentration and market growth rates. Although we could not establish the standard result that growth rates will fall with a lower concentration, we were able to do so for Cournot competition at low enough concentration levels. Still, a reverse result remains possible for both Bertrand and Cournot competition at high levels of concentration close to duopoly.

It should be noted that most of the effects we consider disappear with a large number of firms. Likewise, some of the results must be modified if we consider free-entry into the hi-tech industry. Nevertheless, we argue that in practice the barriers to entering such industries are very high and often fall under state regulation, e.g. by way of licensing. We believe that these circumstances keep the number of incumbent hi-tech firms small enough for the effects we describe to be of realistic magnitudes.

In matters of policy, our results suggest that when the number of firms in the hi-tech industry is influenced by government policy and firms engage in relatively mild competition (as identified by the size of mark-ups), a policy that aims at creating more competition in order to bolster growth may in fact be counterproductive. This concern relates to policies that attempt to facilitate entry into 
industry as well as other measures focused on lowering mark-ups. However, licensing, which restricts the number of competing firms to low numbers, may bring about higher and even socially optimal rates of growth. Our analysis implies that taxation of the traditional sector (or alternatively subsidies, to research) as a means of restoring (constrained) social optimality may not be necessary; in fact, the direct opposite may happen.

Still many important issues remain unresolved. Although our treatment of strategic interactions is not exhaustive, we do find the differential game approach too complicated to offer any practical policy suggestions. We feel that more insight could be gained by partially relaxing the assumption that knowledge spill-overs are completely unintentional, while still keeping the analysis tractable along the lines of this paper. In a companion paper, we head in this direction by assuming that the capacity of a rival firm to absorb the results of the rival's research is endogenous to the proportion of the resources allocated to research.

Further, it seems to us that the framework introduced in this paper could be successfully applied in an international setting to address issues of optimal policy design among exporting or importing companies. The seminal papers on trade and growth (see e.g. Grossman and Helpman, 1990a,b) introduce endogenous growth models into an international setting under very restrictive assumptions that prevent the analysis of more subtle issues underpinning growth and development. In particular, the propagation of technological change across nations was often modelled on an ad hoc basis or was simply given exogenously. Under such conditions, room for analysing the strategic effects of various policy tools 
such as tariff barriers or quantitative protection is confined, and the analysis remains almost exclusively in the domain of strategic trade literature. Our belief that the approach of this paper could help tackle these issues is especially motivated by the close resemblance of our present results to those of strategic trade literature.

\section{References}

[1] Aghion, P., Howitt, P. 1996, Endogenous Growth Theory, MIT Press, Cambridge, MA

[2] Baldwin, R.E., Forslid, R. 1998:" Trade and growth: Any unfinished business", European Economic Review 42

[3] Brander, J.A.: "Strategic trade policy", in: Grossman, G., Rogoff, K., eds, Handbook of International Economics, Vol III, Elsevier, 1995

[4] Dixit, A.K., Stiglitz J.E.:Monopolistic competition and optimum product diversity. American Economic Review 67, 1977.

[5] Grossman, G. M., Helpman, E. 1990a:" Comparative advantage and longrun growth", American Economic Review, 80

[6] Grossman, G. M., Helpman, E. 1990b:"Trade, innovation and growth", American Economic Review, 80

[7] Krugman, P. 1995:"Increasing returns, imperfect competition and the positive theory", in Grossman, G. M., Rogoff, K. (eds.), Handbook of International Economics, Vol. 3, Elsevier Science Amsterdam 
[8] Neary, J.P.,1999, "R\&D in developing countries: what should governments do?", conference paper, World Bank Conference on Development Economics, 21-23 June

[9] Rivera-Batiz, L.A. and Romer, P.M. 1991a:"International trade with endogenous technological change”, European Economic Review, 35

[10] Rivera-Batiz, L.A. and Romer, P.M. 1991b:"Economic integration and endogenou

[11] Romer, P. 1990a:"Endogenous technological change", Journal of Political Economy 84

[12] Romer, P. 1990b:" Are non-convexities important for understanding growth?", American Economic Review 80

[13] Smulders, S., van de Klundert, Th.: Imperfect competition, concentration and growth with firm specific R\&D. European Economic Review 39, 139-60, 1995.

[14] Sutton, J.: Sunk Costs and Market Structure; Price competition, Advertising and Evolution of

Concentration. MIT Press, Cambridge, MA, 1991

[15] Peretto, P.: Sunk Costs, Market Structure, and Growth, International Economic Review, 1996

[16] Vencatachellum, D., 1998, Endogenous growth with strategic interactions, Journal of Economic Dynamics and Control, 23 
[17] Van de Klundert, Th., Smulders, S.: Growth, Competition and Welfare, Scandinavian Journal of Economics 99(1), 1997.

[18] Yang, X. and Heidra, B.J.:Monopolistic competition and optimum product diversity:Comment., American Economic Review 83,1993

\section{A Appendix}

\section{A.1 Appendix 1}

\section{A.1.1 Bertrand competition}

We need to evaluate the expression $\frac{\partial p_{j}\left(h_{j}, \mathbf{h}_{-j}\right)}{\partial h_{i}}$ from (34). Note that $p_{i}=p_{i}(\mathbf{h})=$ $p_{i}\left(h_{i}, \mathbf{h}_{-i}\right)$ is defined in $(21)$ as the result of the second stage competition in prices. This relationship is a reduced form product of the system of F.O.C.s for the following problem:

$$
F_{i}(\mathbf{p}) \equiv \frac{\partial R_{i}(\mathbf{p})}{\partial p_{i}}-\frac{\partial x_{i}(\mathbf{p})}{\partial p_{i}} c\left(h_{i}\right)=0, \forall i=1, \ldots, N
$$

where $R_{i}(\mathbf{p})$ is the current revenue function of firm $i$, i.e. $R_{i}(\mathbf{p})=p_{i} x_{i}(\mathbf{p})$, and $c\left(h_{i}\right)$ is the marginal cost function, defined for this problem as $c\left(h_{i}\right)=\frac{w}{h_{i}}$. With this implicit system, we may compute the derivative $\frac{\partial p_{j}\left(h_{j}, \mathbf{h}_{-j}\right)}{\partial h_{i}}$. Using the implicit function theorem, we may differentiate the system in (72) totally to obtain:

$$
\left(\begin{array}{c}
\nabla\left[\frac{\partial R_{1}(\mathbf{p})}{\partial p_{1}}-\frac{\partial x_{1}(\mathbf{p})}{\partial p_{1}} c\left(h_{1}\right)\right] \\
\cdots \\
\nabla\left[\frac{\partial R_{N}(\mathbf{p})}{\partial p_{N}}-\frac{\partial x_{N}(\mathbf{p})}{\partial p_{N}} c\left(h_{N}\right)\right]
\end{array}\right)(d \mathbf{p})=
$$




$$
=\left(\begin{array}{lll}
\frac{\partial x_{1}(\mathbf{p})}{\partial p_{1}} c^{\prime}\left(h_{1}\right) & \ldots & 0 \\
\ldots & \ldots & \ldots \\
0 & \ldots & \frac{\partial x_{N}(\mathbf{p})}{\partial p_{N}} c^{\prime}\left(h_{N}\right)
\end{array}\right)(d \mathbf{h}) .
$$

The matrix on the left-hand side of the equation is in fact:

$$
\begin{aligned}
A & \equiv\left(\begin{array}{l}
\nabla\left[\frac{\partial R_{1}(\mathbf{p})}{\partial p_{1}}-\frac{\partial x_{1}(\mathbf{p})}{\partial p_{1}} c\left(h_{1}\right)\right] \\
\ldots \\
\nabla\left[\frac{\partial R_{N}(\mathbf{p})}{\partial p_{N}}-\frac{\partial x_{N}(\mathbf{p})}{\partial p_{N}} c\left(h_{N}\right)\right]
\end{array}\right) \\
& =\left(\begin{array}{lll}
\frac{\partial F_{1}(\mathbf{p})}{\partial p_{1}} & \ldots & \frac{\partial F_{1}(\mathbf{p})}{\partial p_{N}} \\
\ldots & \ldots & \ldots \\
\frac{\partial F_{N}(\mathbf{p})}{\partial p_{1}} & \ldots & \frac{\partial F_{N}(\mathbf{p})}{\partial p_{N}}
\end{array}\right)
\end{aligned}
$$

However, because we focus on symmetric equilibria, the functional symmetry of demand with respect to rival firms' prices (noted in the main text) enables us to distinguish only own and cross price derivatives. Denoting $i$ as any firm and $j$ as any firm other than $i$, we may rewrite matrix $A$ in the following form:

$$
\begin{aligned}
A & =\left(\begin{array}{llll}
a & b & \ldots & b \\
b & a & \ldots & b \\
\ldots & \ldots & \ldots & \ldots \\
b & b & \ldots & a
\end{array}\right) \\
a & =\frac{\partial F_{i}(\mathbf{p})}{\partial p_{i}}=2 \frac{\partial x_{i}(\mathbf{p})}{\partial p_{i}}+\frac{\partial^{2} x_{i}(\mathbf{p})}{\left(\partial p_{i}\right)^{2}}\left(p_{i}-c\left(h_{i}\right)\right) \\
b & =\frac{\partial F_{i}(\mathbf{p})}{\partial p_{j}}=\frac{\partial x_{i}(\mathbf{p})}{\partial p_{j}}+\frac{\partial^{2} x_{i}(\mathbf{p})}{\partial p_{i} \partial p_{j}}\left(p_{i}-c\left(h_{i}\right)\right) .
\end{aligned}
$$

This form of matrix A is particularly useful, since its determinant can be readily computed. From the functional symmetry, we also know that in a symmetric equilibrium $\frac{\partial p_{j}\left(h_{j}, \mathbf{h}_{-j}\right)}{\partial h_{i}}=\frac{\partial p_{i}\left(h_{j}, \mathbf{h}_{-j}\right)}{\partial h_{j}}$. Hence, in system (73) we may denote the first row as corresponding to the $i$ th firm and the last row as corresponding to the $j$ th firm. This allows us to study the effects of a variation in $h_{j}$ on $p_{i}$ using a modified system: 


$$
\left(\begin{array}{lll}
a & \ldots & b \\
\ldots & \ldots & \ldots \\
b & \ldots & a
\end{array}\right)\left(\begin{array}{l}
\frac{\partial p_{i}(\mathbf{h})}{\partial h_{j}} \\
\ldots \\
\frac{\partial p_{j}(\mathbf{h})}{\partial h_{j}}
\end{array}\right)=\left(\begin{array}{c}
0 \\
\ldots \\
\frac{\partial x_{j}(\mathbf{p})}{\partial p j} c^{\prime}\left(h_{j}\right)
\end{array}\right) .
$$

Invoking Crammer's rule ${ }^{16}$, we obtain

$$
\frac{\partial p_{i}(\mathbf{h})}{\partial h_{j}}=\frac{a+(N-2) b}{a^{2}+(N-2) b a-(N-1) b^{2}} \frac{\partial x_{j}(\mathbf{p})}{\partial p_{j}} c^{\prime}\left(h_{j}\right)
$$

where $N$ denotes the number of rows in matrix $A$ (equal to the number of firms). Substituting for $a$ and $b$ from above and for $p_{i}$ from (17), we receive after computing the partial derivatives the following expression:

$$
\begin{aligned}
\frac{\partial p_{j}\left(h_{j}, \mathbf{h}_{-j}\right)}{\partial h_{i}}= & \frac{\partial p_{i}(\mathbf{h})}{\partial h_{j}}=-\frac{w}{h_{i}^{2}} d^{B}, \\
d^{B}= & e^{B} \frac{(\epsilon N+1-\epsilon)(\epsilon-1)}{N\left[e^{B}(\epsilon(N-1)+1+N)+2(\epsilon(1-N)-1)\right]} \ldots \\
& \frac{\left[N\left(\epsilon-e^{B}\right)+2(1-\epsilon)\right]}{\left[e^{B}(\epsilon(1-2 N)-1)+\epsilon^{2}(N-2)+\epsilon(N+2)\right]} .
\end{aligned}
$$

If we now substitute expression (20) for the perceived price elasticity for Bertrand competition, we obtain the expression that appears in the main text.

$$
d^{B}=\frac{\epsilon(N-1)+1}{(N-1)\left(\epsilon N^{2}-\epsilon(N-1)-1\right)}>0 .
$$

\section{A.1.2 Cournot competition}

We need to evaluate the expression $\frac{\partial x_{j}(\mathbf{h})}{\partial h_{i}}$ from (73). Note that $x_{i}=x_{i}(\mathbf{h})=$ $x_{i}\left(h_{i}, \mathbf{h}_{-i}\right)$ is defined in (21) as the result of second stage competition in quantities. This relationship is a reduced form product of the system of F.O.C.s for this problem:

\footnotetext{
${ }^{16}$ Details about the computation of matrix determinants involved in Crammer's rule can be obtained from the author upon request.
} 


$$
G_{i}(\mathbf{x}) \equiv \frac{\partial R_{i}(\mathbf{x})}{\partial x_{i}}-c\left(h_{i}\right)=0, \forall i=1, \ldots, N
$$

where $R_{i}(\mathbf{x})$ is the current revenue function of firm $i$, i.e. $R_{i}(\mathbf{x})=p_{i}(\mathbf{x}) x_{i}$, and $c\left(h_{i}\right)$ is the marginal cost function, defined for this problem as $c\left(h_{i}\right)=\frac{w}{h_{i}}$. With this implicit system, we may compute the derivative $\frac{\partial x_{j}(\mathbf{h})}{\partial h_{i}}$. Using the implicit function theorem, we may differentiate the system in (76) totally to obtain:

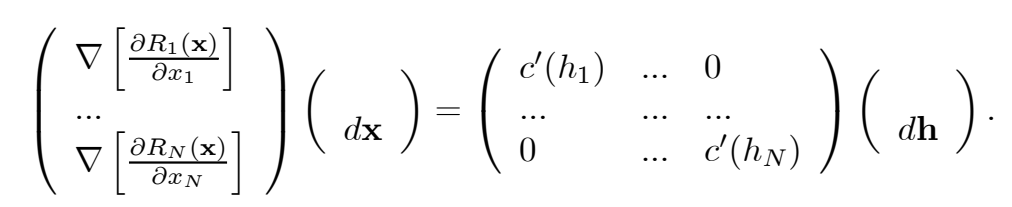

The matrix on the left-hand side of the equation is in fact:

$$
\left(\begin{array}{l}
\nabla\left[\frac{\partial R_{1}(\mathbf{x})}{\partial x_{1}}\right] \\
\ldots \\
\nabla\left[\frac{\partial R_{N}(\mathbf{x})}{\partial x_{N}}\right]
\end{array}\right)=\left(\begin{array}{lll}
\frac{\partial G_{1}(\mathbf{x})}{\partial x_{1}} & \ldots & \frac{\partial G_{1}(\mathbf{x})}{\partial x_{N}} \\
\ldots & \ldots & \ldots \\
\frac{\partial G_{N}(\mathbf{x})}{\partial x_{1}} & \ldots & \frac{\partial G_{N}(\mathbf{x})}{\partial x_{N}}
\end{array}\right)
$$

However, because we are interested in symmetric equilibria, the functional symmetry of demand with respect to the rival firm's prices (noted in the main text) enables us to distinguish only own and cross price derivatives. Denoting $i$ as any firm and $j$ as any firm other than $i$, we may therefore rewrite this matrix in the following form:

$$
\begin{aligned}
A & =\left(\begin{array}{cccc}
a & b & \ldots & b \\
b & a & \ldots & b \\
\ldots & \ldots & \ldots & \ldots \\
b & b & \ldots & a
\end{array}\right) \\
a & =\frac{\partial G_{i}(\mathbf{x})}{\partial x_{i}}=2 \frac{\partial p_{i}(\mathbf{x})}{\partial x_{i}}+\frac{\partial^{2} p_{i}(\mathbf{x})}{\left(\partial x_{i}\right)^{2}} x_{i} \\
b & =\frac{\partial G_{i}(\mathbf{x})}{\partial x_{j}}=\frac{\partial p_{i}(\mathbf{x})}{\partial x_{j}}+\frac{\partial^{2} p_{i}(\mathbf{x})}{\partial x_{i} \partial x_{j}} x_{i}
\end{aligned}
$$


From the functional symmetry, we also know that in a symmetric equilibrium $\frac{\partial x_{j}(\mathbf{h})}{\partial h_{i}}=\frac{\partial x_{i}(\mathbf{h})}{\partial h_{j}}$. Hence, in system (77) we may denote the first row as corresponding to the $i$ th firm and the last row as corresponding to the $j$ th firm. This allows us to study the effects of a variation in $h_{j}$ on $x_{i}$ using a modified system:

$$
\left(\begin{array}{lll}
a & \ldots & b \\
\ldots & \ldots & \ldots \\
b & \ldots & a
\end{array}\right)\left(\begin{array}{l}
\frac{\partial x_{i}(\mathbf{h})}{\partial h_{j}} \\
\ldots \\
\frac{\partial x_{j}(\mathbf{h})}{\partial h_{j}}
\end{array}\right)=\left(\begin{array}{c}
0 \\
\ldots \\
c^{\prime}\left(h_{j}\right)
\end{array}\right)
$$

Invoking Crammer's rule ${ }^{17}$, we obtain

$$
\frac{\partial x_{i}(\mathbf{h})}{\partial h_{j}}=\frac{a+(N-2) b}{a^{2}+(N-2) b a-(N-1) b^{2}} c^{\prime}\left(h_{j}\right),
$$

where $N$ denotes the number of rows in the matrix (equal to the number of firms). Substituting for $a$ and $b$ from above and computing the partial derivatives herein, we receive the expression that appears in the text:

$$
\frac{\partial x_{j}(\ldots)}{\partial h_{i}}=\frac{\partial x_{i}(\mathbf{h})}{\partial h_{j}}=-\frac{w}{h_{i}^{2}} \frac{x_{i}}{p_{i}} d^{C}, d^{C}=\frac{\epsilon(N-2)}{(N-1)(\epsilon+N-2)} \geq 0
$$

\section{A.2 Appendix 2}

The functional forms of the expressions $A^{C}(N, \epsilon)=1+\frac{(\epsilon-1)(N-2)}{N(\epsilon+N-2)}$ and $A^{B}(N, \epsilon)=$ $1-\frac{\epsilon-1}{\epsilon N^{2}-\epsilon(N-1)-1}=\frac{\epsilon N(N-1)}{\epsilon N^{2}-\epsilon(N-1)-1}$ make it difficult to analyse their behaviour on a particular domain of interest, i.e. $\epsilon>1$ and $N \geq 2$. Hence, we rely on numerical simulations, shown in Figure 1, in order to support our intuition.

First, it is immediately seen that $A^{C}(N, \epsilon)>1>A^{B}(N, \epsilon)$ on this parameter space. Second, the limit of these expressions for $N \rightarrow \infty$ is indeed 1 , for every

\footnotetext{
${ }^{17}$ Details about the computation of matrix determinants involved in Crammer's rule can be obtained from the author upon request.
} 
$\epsilon$. Similarly, the behaviour of the expressions at the boundary value of $N=2$ can be readily established: $A^{C}(N=2, \epsilon)=1, \lim _{\epsilon \rightarrow \infty} A^{B}(N=2, \epsilon)=2 / 3$. However, the behaviour inside the domain is quite different: while $A^{B}(N, \epsilon)$ is monotonic with respect to $N$ (for a given $\epsilon$ ), $A^{C}(N, \epsilon)$ generally is not, as the following partial derivatives reveal:

$$
\begin{aligned}
& \frac{\partial A^{B}(N, \epsilon)}{\partial N}=\frac{\epsilon(\epsilon-1)(2 N-1)}{\left(\epsilon N^{2}+\epsilon-N \epsilon-1\right)^{2}}>0 \\
& \frac{\partial A^{C}(N, \epsilon)}{\partial N}=\frac{(\epsilon-1)\left(-N^{2}+4 N+2 \epsilon-4\right)}{N^{2}(\epsilon+N-2)^{2}} .
\end{aligned}
$$

From inspecting the latter expression, we can establish that $A^{C}(N, \epsilon)$ falls in $N$ for $N$ larger or equal to a critical value $\check{N}=2+\sqrt{2 \epsilon}$, which is obtained by setting the partial derivative to zero and ignoring the irrelevant part of the solution. We may infer, then, that the strategic term $A^{C}(N, \epsilon)$ reaches its maximum at values of $N$ larger but relatively close to 3 . Indeed, Figure 1 demonstrates that for plausible values of $\epsilon$ the strategic interactions have their greatest impact at relatively high levels of concentration. On the other hand, they have no impact with duopoly or when the number of firms is infinitely large.

\section{A.3 Appendix 3}

A direct comparison of growth rates under the two modes of competition is cumbersome, so to better probe the effect of competition on constrained growth rates, we must weigh the two opposite effects (toughness of competition and strategic interactions) in expressions (63) and (64) in a particular way. We 
choose a first order Taylor approximation as one possibility, assuming that it can give us reasonable intuition as long as the rates under the two modes of competition are not too far apart. Our basic function to be approximated is the general function for a constrained growth rate in (59):

$$
g(l)=\frac{\gamma \sigma\left(e^{l}-1\right)\left(\frac{L}{N}-f\right) A^{l}-\theta\left(e^{l}-\sigma\right)}{\alpha\left(e^{l}-\sigma\right)+A \sigma\left(e^{l}-1\right)}, l=B, C .
$$

Because evaluation of this function at the two points representing Bertrand and Cournot modes of competition makes comparison difficult, we approximate the growth rate for Bertrand at the point of Cournot. In other words, we approximate the value of $g(B)$ as the first order Taylor expansion of $g(l)$ at $l=C$. Then we obtain for the approximate difference between the two rates as:

$$
g(C)-g(B) \simeq \frac{\partial g^{C}(N, e, A)}{\partial A}\left(A^{C}-A^{B}\right)-\frac{\partial g^{C}(N, e, A)}{\partial e}\left(e^{B}-e^{C}\right) .
$$

The first term corresponds to the impact of strategic effects (making Cournot growth rates higher), while the second reflects the toughness of the competition effect (favouring Bertrand). The differences between the strategic variables $A^{C}-A^{B}$ and perceived price elasticities $e^{B}-e^{C}$ thus provide reasonable weights for the two opposite effects. Substituting for the partial derivatives from (63) and (64), we obtain:

$$
\begin{aligned}
& g(C)-g(B) \simeq \\
& \frac{\gamma \alpha\left(\frac{L}{N}-f\right)+\theta}{\left(\alpha\left(e^{C}-\sigma\right)+A^{C} \sigma\left(e^{C}-1\right)\right)^{2}} \sigma\left[\begin{array}{c}
\left(e^{C}-1\right)\left(e^{C}-\sigma\right)\left(A^{C}-A^{B}\right) \\
-A^{C}(1-\sigma)\left(e^{B}-e^{C}\right)
\end{array}\right] .
\end{aligned}
$$


Let's denote the term inside the square brackets as $Z$. The sign of this term, which is a nontrivial function of two model parameters $(\epsilon, \sigma)$ and a number of firms $N$, determines the relationship between $g(C)$ and $g(B)$, because the preceding terms are assumed to be positive. The parameter upon which the relationship is likely to depend is the share of hi-tech goods in consumption $\sigma$. We thus compute the critical value of $\sigma$ at which the expression equals zero:

$$
\widetilde{\sigma}=\frac{A^{C}\left(e^{B}-e^{C}\right)-\left(e^{C}-1\right) e^{C}\left(A^{C}-A^{B}\right)}{A^{C}\left(e^{B}-e^{C}\right)-\left(e^{C}-1\right)\left(A^{C}-A^{B}\right)} .
$$

As long as $\widetilde{\sigma} \leq 1$, we can expect Cournot competition to yield higher rates of growth for any $\sigma>\widetilde{\sigma}$, because we know that for $\sigma=1$, it is always so. Because $\widetilde{\sigma}$ is a function of $\epsilon$ and $N$ only, we may study its behaviour along these two dimensions. It is possible to show that the critical value rises with both the elasticity of substitution (and approaches unity for large $\epsilon$ ) and the level of concentration (approaches minus infinity for large $N$ ) in our domain of interest. These properties are demonstrated with the help of numerical simulations in Figure 2. The behaviour of $\epsilon$ is intuitive, because almost perfect substitutability reduces the scope for strategic concentration, favouring Bertrand growth rates.

Not so straightforward is the behaviour of $\widetilde{\sigma}$ with respect to $N$. It appears from Figure 2 that $\widetilde{\sigma}$ not only falls in $N$, but does so very rapidly. Interestingly, at lower levels of concentration we observe that Cournot competition delivers higher constrained rates of growth, almost irrespective of the degree of substitutability. This is unusual, since one might expect that at high levels of concentration where the strategic interactions are unimportant, the effect of lower monopoly power under Bertrand would dominate. Yet, it turns out that the 
difference between the perceived price elasticities $\left(e^{B}-e^{C}=\frac{(\epsilon-1)^{2}(N-1)}{N(N+\epsilon-1)}\right)$ vanishes with higher $N$ even more rapidly than the difference between the strategic terms $\left(A^{C}-A^{B}=\frac{(\epsilon-1)(N-2)}{N(\epsilon+N-2)}+\frac{\epsilon-1}{\epsilon N^{2}-\epsilon(N-1)-1}\right)$.

From the graphs in Figure 2, it becomes apparent that differentiability is important for determining $\widetilde{\sigma}$ only at concentrations close to duopoly. For realistically differentiated markets (with values of $\epsilon$ up to 5), competition of as few as 5 companies and a relatively low share of differentiated goods in consumption are required for dominating Bertrand growth rates.

\section{A.4 Appendix 4}

In this appendix, we provide further information about the coincidence of the market and social constrained equilibria under Cournot competition. The level of concentration at which this coincidence occurs is given by condition (70):

$$
\frac{(\epsilon-1)(N-2)}{N(\epsilon+N-2)}=\alpha \frac{g^{S P}(N)}{\theta} .
$$

Instead of solving this cubic equation in $N$, we characterise the solution indirectly, using mainly graphical tools. We denote the LHS of the condition as $G(N)$ and use expression (68) to substitute for the socially optimal rate of growth:

$$
G(N)=\frac{\alpha \gamma L}{\theta N}-\frac{\alpha}{\theta} \gamma f-\theta \equiv \frac{a}{N}-b .
$$

Because we want to place some restrictions on the model parameters, we choose the substitutions of $a$ and $b$ for the various model parameters in order 
to characterise these restrictions in a parsimonious way. Even though $a$ and $b$ contain some common elements, there are always enough free parameters to think of them as independent. The most obvious restriction that we can place on model parameters is the requirement that $g^{S P}(N)$ be positive for reasonable levels of concentration. This requirement stems from the observation that market long-run growth rates are typically positive and that socially optimal growth rates tend to exceed market ones. For our new parametrisation, this implies $\frac{a}{b}>N$, for a range of $N$ where we find such a restriction reasonable. Because of the linearity of this relationship, as $N$ increases from 2 or 3 , the value of $b$ quickly becomes unimportant in relation to $a$. This allows us, at least for the moment, to ignore $b$ in condition (81) which thus results in the following approximation:

$$
G(N) \simeq \frac{a}{N}
$$

Unlike the original condition in (70), this equation can be solved relatively easily for a single solution:

$$
N=2+\frac{a \epsilon}{\epsilon-1-a} .
$$

The solution exists on the plausible domain of $N \geq 2$ only under specific circumstances, namely for high $\epsilon$ and low $a$, making the coincidence of social and marker equilibria fairly unlikely. In other words, a social optimum will be attained only if hi-tech goods are sufficiently good substitutes $(\epsilon \geq 1+a)$, which is quite reasonable. Because these conclusions hold only in approximation ig- 
noring term $b$ in (81), we support our intuition through a graphic representation of condition (82) in Figure 3. There we plot separately the left- and right-hand sides of the condition as functions of $N$ for several parameter values of $\epsilon$, and $a$. Apparently, the non-monotonic plots of $G(N)$ scale upward for larger $\epsilon$, while hyperbolic plots of $a / N$ scale downward with lower $a$. The coincidence of market and social constrained rates of growth arise where the two types of curves intersect. From the figure, we observe that for any given $a$, the two curves will always intersect for $\epsilon$ high enough. Because $b$ is the long-run limit of the right-hand side expression in (81) as $N \rightarrow \infty$, its inclusion would shift the corresponding hyperbolic curve down in Figure 3, lowering $N$ where the coincidence may occur.

\section{A.5 Appendix 5}

In this appendix we show how the results in the main text would change if we considered strategic interactions based on the existence of knowledge spillovers. This channel rests on the recognition that a firm's stock of firm specific knowledge is in fact dependent on the knowledge stock accumulated by each of its rivals through the industry average stock of knowledge. For instance $h_{i}\left(\mathbf{h}_{-i}\right)$,

because $H=\frac{\sum_{l} h_{l}}{N}$. The inclusion of this strategic channel would call for a more general treatment of condition $\frac{\partial H_{i}^{B, C}}{\partial h_{i}}=-\dot{\lambda}_{i}$ characterising the intertemporal allocation of resources between research and production within hi-tech firms. We will therefore investigate the necessary modifications of this condition for each competition mode. 


\section{A.5.1 Bertrand competition}

Intertemporal condition (33) including the spill-over effect look as follows:

$$
\begin{gathered}
\frac{\partial H_{i}^{B}}{\partial h_{i}}= \\
e^{-\int_{t}^{s} r_{\tau} d \tau}\left[\begin{array}{c}
\nabla x_{i}(\mathbf{p})\left(\begin{array}{c}
\frac{\partial p_{i}\left(h_{i}, \mathbf{h}_{-i}\right)}{\partial h_{i}} \\
\frac{d \mathbf{p}_{-i}\left(h_{i}, \mathbf{h}_{-i}\right)}{d h_{i}}
\end{array}\right)\left(p_{i}(\mathbf{h})-\frac{w}{h_{i}}\right)+\ldots \\
\ldots+x_{i}(\mathbf{p})\left(\frac{\partial p_{i}\left(h_{i}, \mathbf{h}_{-i}\right)}{\partial h_{i}}+\frac{w}{h_{i}^{2}}\right)
\end{array}\right]+ \\
+\quad \lambda_{i} \gamma\left(\frac{H}{h_{i}}\right)^{\alpha} L_{I_{i}}\left[(1-\alpha)+\frac{\alpha^{2}}{N}\right]=-\dot{\lambda}_{i} .
\end{gathered}
$$

The condition has changed in two respects. First, the last term before the equation sign features $\left[(1-\alpha)+\frac{\alpha^{2}}{N}\right]$ instead of $(1-\alpha)$. The difference is the extra effect of a firm's own R\&D investment on the speed of R\&D accumulation through its impact on the industry average. The extra effect is, however, of the third order, even at high levels of concentration, provided the extent of spillovers $(\alpha)$ remains reasonably small. As the growth engine of this model rests on accumulation of firm specific knowledge, we assume $\alpha$ to be small enough to ignore $\frac{\alpha^{2}}{N}$ term. This assumption lets us preserve all other computations performed in the main text save those leading up to conditions (35) or (39) which we compute below.

As a second modification of condition (33), the vector of partial derivatives $\frac{\partial \mathbf{p}_{-i}\left(h_{i}, \mathbf{h}_{-i}\right)}{\partial d h_{i}}$ featured in (33) has been replaced by the corresponding vector of total derivatives $\frac{d \mathbf{p}_{-i}\left(h_{i}, \mathbf{h}_{-i}\right)}{d h_{i}}$, because each element of vector $\mathbf{h}_{-i}$ is a function of $h_{i}$ as well. Performing this total derivative, we obtain:

$$
\frac{d \mathbf{p}_{-i}\left(h_{i}, \mathbf{h}_{-i}(\mathbf{h})\right)}{d h_{i}}=\frac{\partial \mathbf{p}_{-i}\left(h_{i}, \mathbf{h}_{-i}\right)}{\partial h_{i}}+\nabla \mathbf{p}_{-i}\left(\mathbf{h}_{-i}\right)\left(\frac{\partial \mathbf{h}_{-i}(\mathbf{h})}{\partial h_{i}}\right),
$$


where the matrix of gradients $\nabla \mathbf{p}_{-i}\left(\mathbf{h}_{-i}\right)$ contains only derivatives of pricing functions with respect to elements of the vector $\mathbf{h}_{-i}$. Consider now a representative element of this vector in a symmetric equlibrium:

$$
\begin{aligned}
\frac{d p_{j \neq i}\left(h_{i}, \mathbf{h}_{-i}(\mathbf{h})\right)}{d h_{i}} & =\frac{\partial p_{j \neq i}\left(h_{i}, \mathbf{h}_{-i}\right)}{\partial h_{i}}+\nabla p_{j \neq i}\left(\mathbf{h}_{-i}\right)\left(\frac{\partial \mathbf{h}_{-i}(\mathbf{h})}{\partial h_{i}}\right) \\
& =\frac{\partial p_{j \neq i}\left(h_{i}, \mathbf{h}_{-i}\right)}{\partial h_{i}}+\frac{\partial p_{j \neq i}\left(\mathbf{h}_{-i}\right)}{\partial h_{j}} \frac{\partial h_{j}(\mathbf{h})}{\partial h_{i}}+(N-2) \frac{\partial p_{j \neq i}\left(\mathbf{h}_{-i}\right)}{\partial h_{l \neq i, j}} \frac{\partial h_{l \neq i, j}(\mathbf{h})}{\partial h_{i}} \\
& =\frac{\partial p_{j \neq i}\left(h_{i}, \mathbf{h}_{-i}\right)}{\partial h_{i}}\left(1+(N-2) \frac{\partial h_{l \neq i, j}(\mathbf{h})}{\partial h_{i}}\right)+\frac{\partial p_{j \neq i}\left(\mathbf{h}_{-i}\right)}{\partial h_{j}} \frac{\partial h_{j}(\mathbf{h})}{\partial h_{i}}
\end{aligned}
$$

In deriving the second and third equalities, we have made use of the fact that $p_{i}\left(h_{i}, \mathbf{h}_{-i}\right)$ is functionally symmetric with respect to the rivals' stocks of knowledge. Hence, the cross derivatives $\left\{\frac{\partial p_{j \neq i}\left(\mathbf{h}_{-i}\right)}{\partial h_{l \neq i, j}}\right\}$ and $\frac{\partial p_{j \neq i}\left(h_{i}, \mathbf{h}_{-i}\right)}{\partial h_{i}}$ are all functionally identical and in a symmetric equilibrium would take on the same value. Functional symmetry is also apparent in $h_{i}\left(h_{-i}\right)$ and so the cross derivatives $\frac{\partial h_{j}}{\partial h_{i \neq j}}$ in vector $\frac{\partial \mathbf{h}_{-i}}{\partial h_{i}}$ would also have the same value, namely $\frac{\partial h_{j}}{\partial h_{i \neq j}}=$ $\alpha \frac{h_{j}}{H} \frac{1}{N}=\frac{\alpha}{N}$. We thus obtain:

$$
\frac{d p_{j \neq i}\left(h_{i}, \mathbf{h}_{-i}(\mathbf{h})\right)}{d h_{i}}=\frac{\partial p_{j \neq i}\left(h_{i}, \mathbf{h}_{-i}\right)}{\partial h_{i}}\left(1+\frac{N-2}{N} \alpha\right)+\frac{\partial p_{j \neq i}\left(\mathbf{h}_{-i}\right)}{\partial h_{j}} \frac{\alpha}{N}
$$

We do observe that in the absence of spill-overs $(\alpha=0)$, the vectors of partial and total derivatives in conditions (33) and (83) coincide. It is also apparent that all rows of gradient $\nabla \mathbf{p}_{-i}\left(\mathbf{h}_{-i}\right)$ have the same functional form, and in the symmetry would be identical. As a corollary, all elements of vector $\frac{d \mathbf{p}_{-i}\left(h_{i}, \mathbf{h}_{-i}\right)}{d h_{i}}$ in (83) share the same functional form as well, so in a symmetric equilibrium would all have the same value. This enables us to treat the vector 
in our subsequent analysis in the same way as we did with vector $\frac{\partial \mathbf{p}_{-i}\left(h_{i}, \mathbf{h}_{-i}\right)}{\partial h_{i}}$ in the main text.

Following the line of argument after condition (33) in the main text, we may simplify condition (83) to obtain the equivalent of (34):

$$
\begin{aligned}
& h_{i}\left[(N-1) \epsilon\left(x_{i} ; p_{j} \mid p_{-j}\right) \frac{x_{i}}{p_{j}} \frac{d p_{j}\left(h_{j}, \mathbf{h}_{-j}\right)}{d h_{i}}\left(p_{i}-\frac{w}{h_{i}}\right)+\frac{w}{h_{i}^{2}}\right]+ \\
+\quad & (1-\alpha) w L_{I_{i}}=\frac{w}{\gamma} \frac{h_{i}^{\alpha}}{H^{\alpha}}\left[-\frac{\dot{w}_{t}}{w_{t}}+(1-\alpha) \frac{\dot{h}_{i, t}}{h_{i, t}}+\alpha \frac{\dot{H}_{t}}{H_{t}}+r_{t}\right] .
\end{aligned}
$$

The only element that needs evaluating is total derivative $\frac{d p_{j}\left(h_{j}, \mathbf{h}_{-j}\right)}{d h_{i}}$ given in (84). To better understand its meaning, we should recall that it represents two channels of strategic interactions. One works through the direct link between investment decisions in the first stage of the game and pricing decisions of rival firms in the second. This, as well as (33) is exemplified by derivative $\frac{\partial p_{j \neq i}\left(h_{i}, \mathbf{h}_{-i}\right)}{\partial h_{i}}$. From the analysis above we know that the effect is negative: investment in $R \& D$ enables lower prices, which triggers a similar reaction of the rival firms. The other channel works indirectly through the impact of R\&D investment on the cost (and so pricing) decisions of the rival firms. It has two components. First, on the impact, R\&D investment of a representative firm lowers the cost functions of the rival firms, who can then charge lower prices as well, keeping prices of other firms unchanged. This is the term $\frac{\partial p_{j \neq i}\left(\mathbf{h}_{-i}\right)}{\partial h_{j}} \frac{\alpha}{N}$, which is negative and is evaluated in Appendix 6. Second, the possibility of lower prices enabled by the lower industry-wide costs triggers a corresponding strategic price response from all the firms before a new static equilibrium is reached. This is 
term $\frac{\partial p_{j \neq i}\left(h_{i}, \mathbf{h}_{-i}\right)}{\partial h_{i}}(N-2) \frac{\alpha}{N}$, which is also negative. By including the knowledge spill-over channel, total derivative $\frac{d p_{j \neq i}\left(h_{i}, \mathbf{h}_{-i}(\mathbf{h})\right)}{d h_{i}}$ unambiguously exceeds in absolute value the partial derivative $\frac{\partial p_{j \neq i}\left(h_{i}, \mathbf{h}_{-i}\right)}{\partial h_{i}}$ considered in the analysis of the main text. As a consequence, the inclusion of this channel increases the impact of strategic interaction on the decisions of investing firms.

Since $\frac{\partial p_{j \neq i}\left(h_{i}, \mathbf{h}_{-i}\right)}{\partial h_{i}}$ has already been evaluated in Appendix 1, the last element to examine is $\frac{\partial p_{j \neq i}\left(\mathbf{h}_{-i}\right)}{\partial h_{j}}$. In Appendix 6, we show that $\frac{\partial p_{j \neq i}\left(\mathbf{h}_{-i}\right)}{\partial h_{j}}=$ $c^{\prime}\left(h_{i}\right) G^{B}$, where $G^{B}$ is a function of the primitive parameters of the model. Because $\frac{\partial p_{j \neq i}\left(\mathbf{h}_{-i}\right)}{\partial h_{j}}$ has the same form as $\frac{\partial p_{j \neq i}\left(h_{i}, \mathbf{h}_{-i}\right)}{\partial h_{i}}=c^{\prime}\left(h_{i}\right) d^{B}$, we can manipulate (85) in much the same way as (34) in the main text to arrive at the analogue of (35):

$$
L_{X_{i}} A^{B}+(1-\alpha) L_{I_{i}}=\frac{1}{\gamma} \frac{h_{i}^{\alpha}}{H^{\alpha}}\left[-\frac{\dot{w}_{t}}{w_{t}}+(1-\alpha) \frac{\dot{h}_{i, t}}{h_{i, t}}+\alpha \frac{\dot{H}_{t}}{H_{t}}+r_{t}\right] .
$$

Although both expressions look equivalent, term $A^{B}$ is a different function of primitive parameters than the same term in (35). This notwithstanding, this term has an indentical economic interpretation as a measure of the extent of strategic interactions. It quantifies the perceived impact of $R \& D$ investment on consumer demand for the production of the investing firm, which arises from the price reaction of its rivals. To see this, note that following the computation which leads to the formulation of (61) we arrive at the following expression for $A^{B}:$

$$
A^{B}=1-(N-1) \frac{\epsilon\left(x_{i} ; p_{j} \mid p_{-j}\right) \epsilon\left(p_{j} ; h_{i}\right)}{1-e^{B}}
$$

where this time, elasticity $\epsilon\left(p_{j} ; h_{i}\right)=\frac{d p_{j}}{d h_{i}} \frac{h_{i}}{p_{j}}$, unlike $\frac{\partial p_{j}}{\partial h_{i}} \frac{h_{i}}{p_{j}}$ previously. The ab- 
solute value of this elasticity is higher than before, because the perceived effects of $\mathrm{R} \& \mathrm{D}$ investment on the pricing decisions of the rival firms include the additional spill-over channel. As a consequence, $A^{B}$ will differ from 1 by more than the same expression considered in the main text (for any given combination of parameters), and thus its impact will be larger. All other analyses of the main text, including the characterisation of the equilibrium and expressions for the constrained growth rate, remain intact. Qualitative conclusions for the Bertrand competition too remain unaffected.

\section{A.5.2 Cournot competition}

We will follow the argumentation in the preceding subsection, and leave out most of the commentary. The analogue of condition (36) can obtained as follows:

$$
\begin{aligned}
& \frac{\partial H_{i}^{C}}{\partial h_{i}}= \\
& e^{-\int_{t}^{s} r_{\tau} d \tau}\left[\frac{\partial x_{i}\left(h_{\left.i, \mathbf{h}_{-i}\right)}\right.}{\partial h_{i}}+x_{i}(\mathbf{h}) \nabla p_{i}(\mathbf{x})\left(\frac{\partial x_{i}\left(h_{i} \mathbf{h}_{-i}\right)}{\partial h_{i}} \frac{\left.d \mathbf{h}_{-i}\right)}{d h_{i}}\right)\right. \\
+\quad & \lambda_{i}(1-\alpha) \gamma\left(\frac{H}{h_{i}}\right)^{\alpha} L_{I_{i}}=-\dot{\lambda}_{i},
\end{aligned}
$$

where again the vector of total derivatives $\frac{d \mathbf{x}_{-i}\left(h_{i}, \mathbf{h}_{-i}\right)}{d h_{i}}$ replaced $\frac{\partial \mathbf{x}_{-i}\left(h_{i,} \mathbf{h}_{-i}\right)}{\partial h_{i}}$ and the effect of R\&D investment on the speed of the firm's own knowledge accumulation process is ignored. A representative element of this vector can be expanded as follows:

$$
\frac{d x_{j}\left(h_{\left.i, \mathbf{h}_{-i}\right)}\right.}{d h_{i}}=\frac{\partial x_{j}\left(h_{i} \mathbf{h}_{-i}(\mathbf{h})\right)}{\partial h_{i}}+\frac{\partial x_{j}\left(h_{i}, \mathbf{h}_{-i}(\mathbf{h})\right)}{\partial h_{j}} \frac{\partial h_{j}(\mathbf{h})}{\partial h_{i}}+\ldots
$$




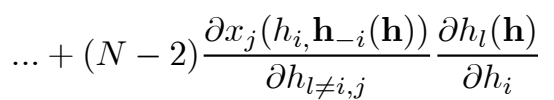

$$
\begin{aligned}
& =\frac{\partial x_{j}\left(h_{i,} \mathbf{h}_{-i}(\mathbf{h})\right)}{\partial h_{i}}\left(1+\frac{N-2}{N} \alpha\right)+\frac{\partial x_{j}\left(h_{i}, \mathbf{h}_{-i}(\mathbf{h})\right)}{\partial h_{j}} \frac{\alpha}{N},
\end{aligned}
$$

where the symmetry in functions and values of the symmetric equilibrium is employed. As in the Bertrand case, the total and partial derivatives of the main text would coincide in the absence of spill-overs.

Following the arguments that led to condition (37) we obtain its analogue:

$$
\begin{gathered}
h_{i} x_{i}(\mathbf{h})\left((N-1) \epsilon\left(p_{i} ; x_{j} \mid \mathbf{x}_{-j}\right) \frac{p_{i}(\mathbf{x})}{x_{j}} \frac{d x_{j}(\mathbf{h})}{d h_{i}}+\frac{w}{h_{i}^{2}}\right)+ \\
+\quad(1-\alpha) w L_{I_{i}}=\frac{w}{\gamma} \frac{h_{i}^{\alpha}}{H^{\alpha}}\left[-\frac{\dot{w}_{t}}{w_{t}}+(1-\alpha) \frac{\dot{h}_{i, t}}{h_{i, t}}+\alpha \frac{\dot{H}_{t}}{H_{t}}+r_{t}\right] .
\end{gathered}
$$

The only element of $\frac{d x_{j}(\mathbf{h})}{d h_{i}}$ in (87) that remains to be evaluated is its own derivative $\frac{\partial x_{j}\left(h_{i}, \mathbf{h}_{-i}(\mathbf{h})\right)}{\partial h_{j}}\left(\frac{\partial x_{j}\left(h_{i} \mathbf{h}_{-i}(\mathbf{h})\right)}{\partial h_{i}}=c^{\prime}\left(h_{i}\right) \frac{x_{i}}{p_{i}} d^{C}\right.$ is evaluated in Appendix 1). In Appendix 6 we demonstrate that $\frac{\partial x_{j}\left(h_{i} \mathbf{h}_{-i}(\mathbf{h})\right)}{\partial h_{j}}=-c^{\prime}\left(h_{i}\right) \frac{x_{i}}{p_{i}} G^{C}>0$, where $G^{C}$ is a function of model parameters only. We observe that this term has the opposite sign from $\frac{\partial x_{j}\left(h_{i}, \mathbf{h}_{-i}(\mathbf{h})\right)}{\partial h_{i}}$. To appreciate the implications of this finding we should recall the two channels of strategic interactions present in $\frac{d x_{j}(\mathbf{h})}{d h_{i}}$. One is the direct link between investment decisions in the first stage of the game and production decisions of rival firms in the second. In (87), as

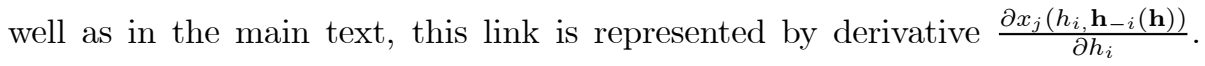
From the analysis above we know that the effect is negative: investment in R\&D enables lower prices, which triggers a corresponding reaction of the rival firms to produce less. The other channel works indirectly through the impact of $\mathrm{R} \& \mathrm{D}$ investment on the cost (and pricing decisions) of the rival firms. It 
has two components. First, on the impact, R\&D investment of a representative firm lowers cost functions of the rival firms, which enables them to produce more at given prices. This is term $\frac{\partial x_{j}\left(h_{i}, \mathbf{h}_{-i}(\mathbf{h})\right)}{\partial h_{j}} \frac{\alpha}{N}$, which is positive and thus works against the effect of the first strategic channel considered in the main text. However, the possibility of higher production (and lower prices), enabled by the lower industry-wide costs, triggers a corresponding strategic response from all the firms who contract their production before a new static equilibrium is reached. This is term $\frac{\partial x_{j}\left(h_{i} \mathbf{h}_{-i}(\mathbf{h})\right)}{\partial h_{i}}(N-2) \frac{\alpha}{N}$, which is negative. By including the knowledge spill-over channel in Cournot competition, then, the relation of total derivative $\frac{d x_{j}(\mathbf{h})}{d h_{i}}$ to the partial one $\frac{\partial x_{j}(\mathbf{h})}{\partial h_{j}}$ is ambiguous. This stands in contrast to the results for Bertrand competition, where the inclusion of this channel strengthened the original results. It still seems fair to conclude, however, that for numbers of firms exceeding 3, even the spill-over channel would likely deliver negative effects and the original results are strengthened.

Because the functional forms of both $\frac{\partial x_{j}\left(h_{i}, \mathbf{h}_{-i}(\mathbf{h})\right)}{\partial h_{j}}$ and $\frac{\partial x_{j}\left(h_{i}, \mathbf{h}_{-i}(\mathbf{h})\right)}{\partial h_{i}}$ in $\frac{d x_{j}(\mathbf{h})}{d h_{i}}$ are similar, we can work with $\frac{d x_{j}(\mathbf{h})}{d h_{i}}$ in much the same way as we did with $\frac{\partial x_{j}(\mathbf{h})}{\partial h_{i}}$. Working along the lines of the main text we observe that condition (39), as well as all other equations in the main text, remain intact:

$$
L_{X_{i}} A^{C}+(1-\alpha) L_{I_{i}}=\frac{1}{\gamma} \frac{h_{i}^{\alpha}}{H^{\alpha}}\left[-\frac{\dot{w}_{t}}{w_{t}}+(1-\alpha) \frac{\dot{h}_{i, t}}{h_{i, t}}+\alpha \frac{\dot{H}_{t}}{H_{t}}+r_{t}\right] .
$$

Although term $A^{C}$ will be a different function of model parameters than the same term in the main text, its interpretation is the same. Like in the Bertrand case, the term measures the perceived impact of $R \& D$ investment on consumer 
demand for the products of the investing firm arising from the price reaction of its rivals. To see this, note that following the computation that lead to the formulation of (62), we arrive at the following expression for $A^{C}$ :

$$
A^{C}=1+(N-1) \frac{e^{C} \epsilon\left(p_{i} ; x_{j} \mid x_{-j}\right) \epsilon\left(x_{j} ; h_{i}\right)}{1-e^{C}},
$$

where $\epsilon\left(x_{j} ; h_{i}\right) \equiv \frac{d x_{j}}{d h_{i}} \frac{h_{i}}{x_{j}}$, unlike $\frac{\partial x_{j}}{\partial h_{i}} \frac{h_{i}}{x_{j}}$ in the main text. The general relation of this newly defined elasticity to its original counterpart is difficult to tell. It seems likely, however, that at low levels of concentration it would exceed the original one. This would enlarge the discrepancy between $A^{C}$ and 1 (for any given combination of parameters) and strengthen the significance of strategic effects in the analysis of the main text.

\section{A.6 Appendix 6}

In this appendix we evaluate the own partial derivatives of choice variable functions with respect to $\mathrm{R} \& \mathrm{D}$ investment, $\frac{\partial p_{j \neq i}\left(\mathbf{h}_{-i}\right)}{\partial h_{j}}$ and $\frac{\partial x_{j}\left(\mathbf{h}_{-i}\right)}{\partial h_{j}}$, as functions of model parameters. The procedure for their evaluation is identical to that evaluating the corresponding cross derivatives in Appendix 1. For this reason, we leave out the discussion and concentrate on the results only.

\section{A.6.1 Bertrand competition}

The derivative of price function with respect to a firm's own R\&D investment can be found in the following equation:

$$
A\left(\begin{array}{l}
\frac{\partial p_{i}(\mathbf{h})}{\partial h_{i}} \\
\ldots \\
\frac{\partial p_{j}(\mathbf{h})}{\partial h_{j}}
\end{array}\right)=\left(\begin{array}{c}
\frac{\partial x_{i}(\mathbf{p})}{\partial p i} c^{\prime}\left(h_{j}\right) \\
0 \ldots \\
0
\end{array}\right),
$$


where matrix $A$ has been defined in the Bertrand part of Appendix 1. Invoking Crammer's rule, we obtain:

$$
\begin{aligned}
\frac{\partial p_{i}(\mathbf{h})}{\partial h_{j}} & =c^{\prime}(h) G^{B} \\
G^{B} & =\frac{(\epsilon N+1-\epsilon)\left(\epsilon N^{2}-2 \epsilon(N-1)+N-2\right)}{(N-1)(\epsilon-1)(\epsilon N(N-1)+\epsilon-1)} .
\end{aligned}
$$

\section{A.6.2 Cournot competition}

The derivative of production with respect to a firm's own $R \& D$ investment can be found in the following equation:

$$
A\left(\begin{array}{l}
\frac{\partial x_{i}(\mathbf{h})}{\partial h_{j}} \\
\ldots \\
\frac{\partial x_{j}(\mathbf{h})}{\partial h_{j}}
\end{array}\right)=\left(\begin{array}{c}
c^{\prime}\left(h_{i}\right) \\
0 \ldots \\
0
\end{array}\right),
$$

where matrix $A$ has been defined in the Cournot part of Appendix 1. Invoking Crammer's rule, we obtain:

$$
\begin{aligned}
\frac{\partial x_{j}\left(\mathbf{h}_{-i}\right)}{\partial h_{j}} & =-c^{\prime}(h) \frac{x_{i}}{p_{i}} G^{C} \\
G^{C} & =\epsilon \frac{-2 \epsilon(N-1)+\epsilon N^{2}+N-2}{(N-1)(\epsilon-1)(N-2+\epsilon)} .
\end{aligned}
$$

\section{B Figures}


Figure 1: Strategic terms

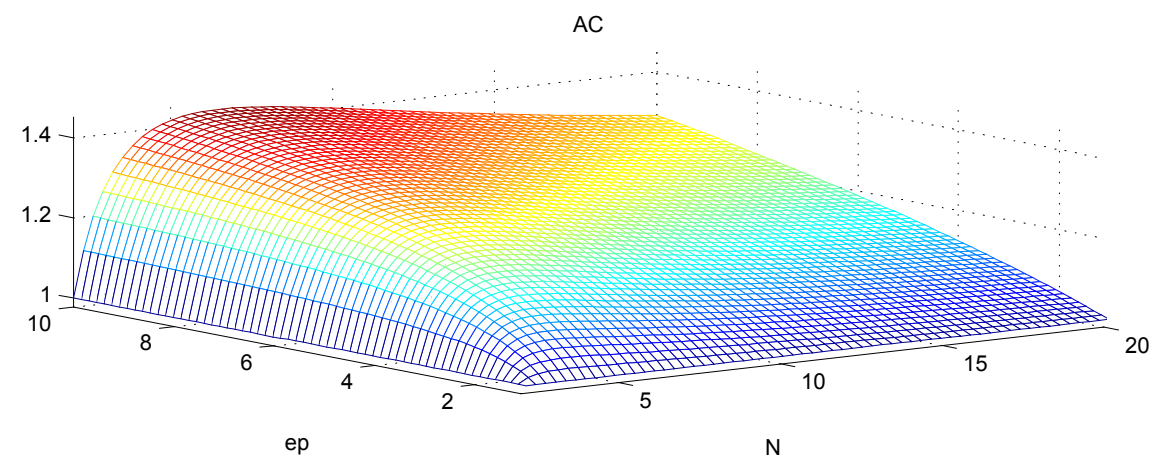

AB

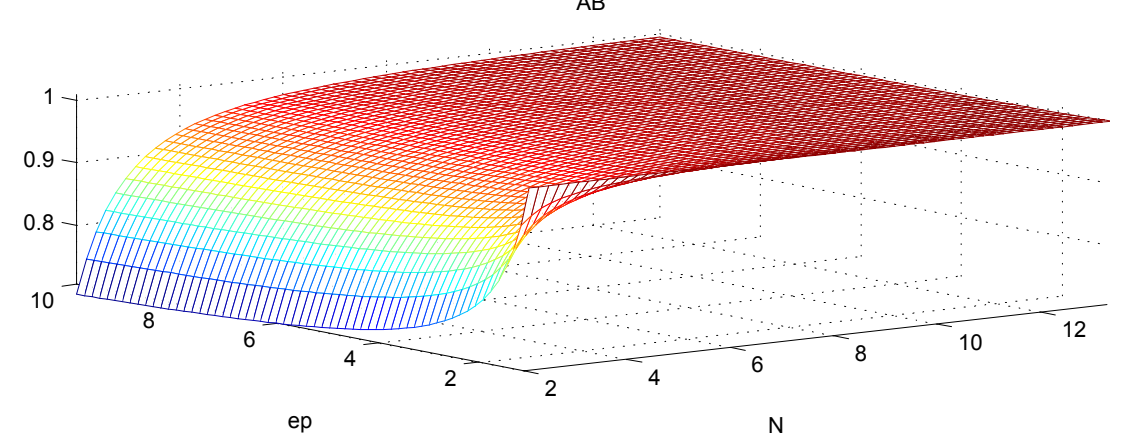


Figure 2: Critical values of $\tilde{\sigma}$
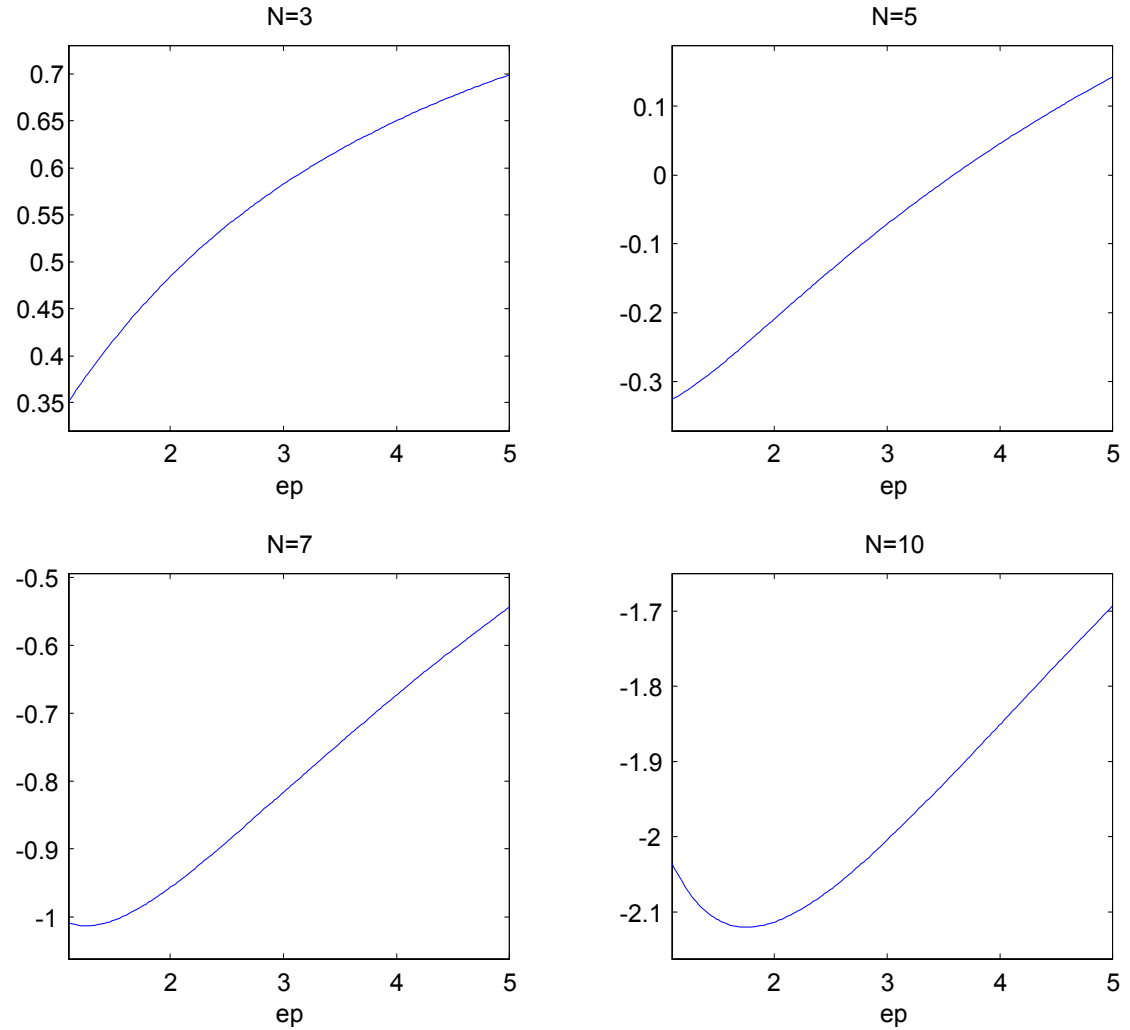
Figure 3: Coincidence of social and market constrained growth rates

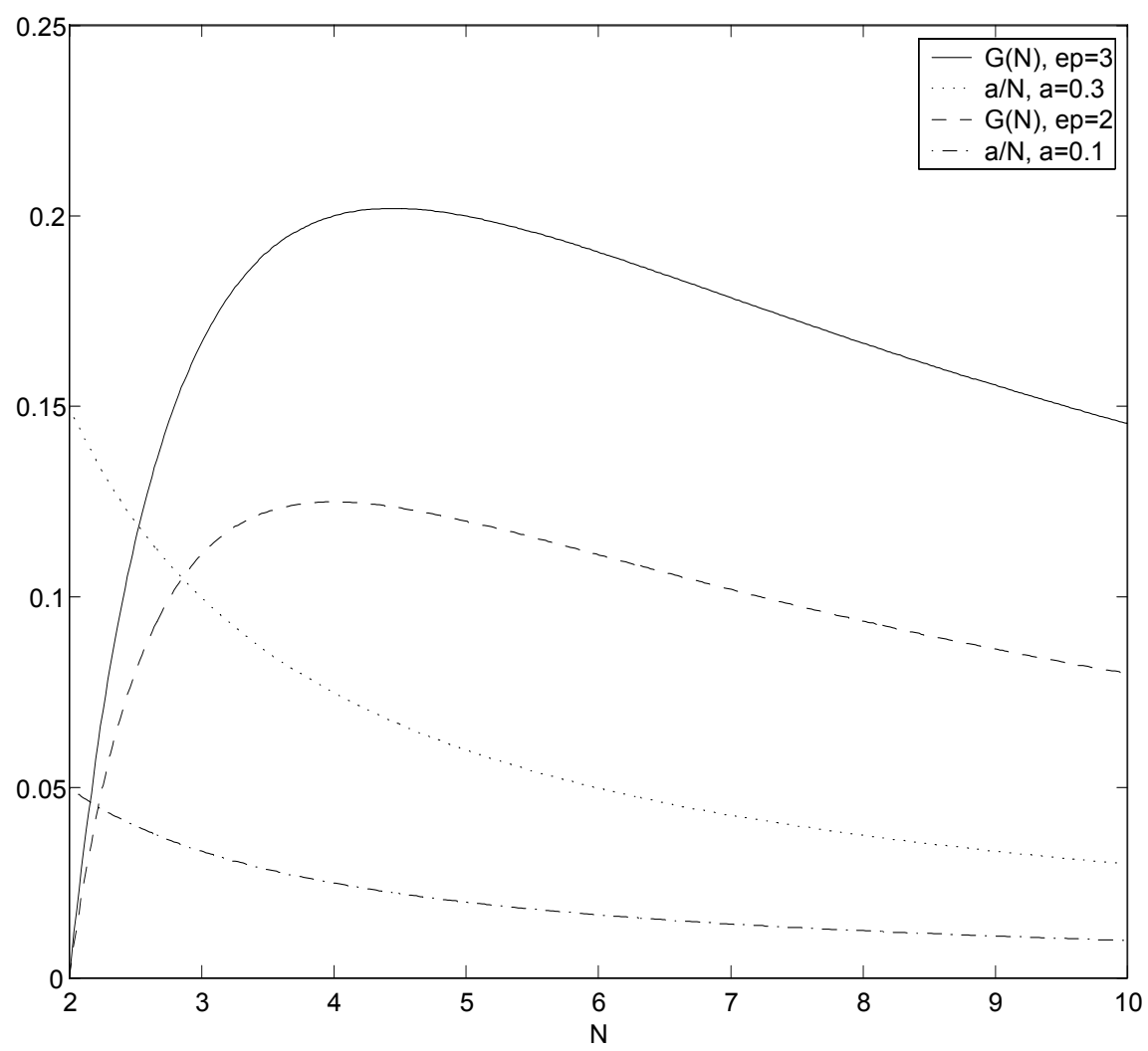

